\title{
Brascamp-Lieb Inequalities on Compact Homogeneous Spaces
}

https://doi.org/10.1515/agms-2019-0007

Received July 9, 2019; accepted August 23, 2019

Abstract: We provide a general strategy to construct multilinear inequalities of Brascamp-Lieb type on compact homogeneous spaces of Lie groups. As an application we obtain sharp integral inequalities on the real unit sphere involving functions with some degree of symmetry.

Keywords: Heat flow; Homogeneous spaces; Multilinear inequalities

MSC: 43A15, 43A $85,52 \mathrm{~A} 40$

\section{Introduction}

Many well-known multilinear inequalities commonly used in analysis, such as multilinear Hölder's inequality, Loomis-Whitney inequality and the sharp Young convolution inequality, can be seen as instances of a broader family of estimates: the so called Brascamp-Lieb inequalities. These are inequalities of the form

$$
\int_{\mathbb{R}^{n}} \prod_{j=1}^{m} f_{j}\left(B_{j} x\right) d x \leq C \prod_{j=1}^{m}\left\|f_{j}\right\|_{L^{p_{j}}\left(\mathbb{R}^{n_{j}}\right)},
$$

where $p_{j} \in[1, \infty], B_{j}: \mathbb{R}^{n} \rightarrow \mathbb{R}^{n_{j}}$ are linear surjective maps and the functions $f_{j}: \mathbb{R}^{n_{j}} \rightarrow \mathbb{R}^{+}$are measurable, for $j=1, \ldots, m$. The constant $C$ in (1.1) is the smallest constant, either finite or infinite, over all measurable inputs $f_{j}$ for which (1.1) holds. This constant depends on the maps $B_{j}$ and the exponents $p_{j}$ and is called the Brascamp-Lieb constant.

These inequalities were extensively studied in the last years, starting from the works of Rogers [20] Brascamp, Lieb and Luttinger [7] and Brascamp and Lieb [6], where the authors studied the rank-one case, that is the case where $n_{j}=1$ for all $j$, using rearrangement techniques. In particular they proved that the BrascampLieb constant is the same if one restricts the inputs to Gaussians, a result known as Lieb's Theorem. This result was then extended to the higher rank case by Lieb in [15], then Barthe gave an alternative proof using transportation of mass techniques in [2].

Another approach to the problem was introduced by Carlen, Lieb, Loss who used heat flow methods to prove Lieb's Theorem in the rank one case in [9]. This approach was rediscovered independently and used by Bennett, Carbery, Christ and Tao to prove Lieb's Theorem in the general case in [5]. In particular they were able to prove the following theorem.

Theorem 1.1 ([5]). The constant $C$ in (1.1) is finite if and only if the scaling condition

$$
\sum_{j=1}^{m} p_{j}^{-1} n_{j}=n
$$

\footnotetext{
^Corresponding Author: Roberto Bramati: Università di Padova, Padova, Italy, E-mail: roberto.bramati@unipd.it
} 
and the dimension condition

$$
\operatorname{dim}(V) \leq \sum_{j=1}^{m} p_{j}^{-1} \operatorname{dim}\left(B_{j} V\right),
$$

for all subspaces $V \subseteq \mathbb{R}^{n}$, are satisfied.

The heat flow technique consists in studying the monotonicity properties of a certain function, depending on a nonnegative parameter that can be thought of as time, that is related to the heat evolution of some functions. Comparing this function at different times is a way of producing inequalities. For example in [5] the authors study, among other things, the case of the so called geometric Brascamp-Lieb inequality (already studied by Ball in [1] and Barthe in [2]), in which the linear maps $B_{j}^{\star}$ are isometries and the condition

$$
\sum_{j=1}^{m} p_{j}^{-1} B_{j}^{\star} B_{j}=\operatorname{Id}_{\mathbb{R}^{n}}
$$

holds. They show that for nonnegative Schwartz functions $f_{j}$ the quantity

$$
Q(t)=\int_{\mathbb{R}^{n}} \prod_{j=1}^{m} u_{j}(t, x) d x
$$

is nondecreasing, where $u_{j}(t, x)^{p_{j}}$ is the solution of the heat equation in $\mathbb{R}^{n}$ with initial datum $f_{j}^{p_{j}} \circ B_{j}$. Inequality (1.1) is then obtained by comparing $\lim _{t \rightarrow 0} Q(t)$ with $\lim _{t \rightarrow \infty} Q(t)$.

In this paper we will interpret inequality (1.1) in the following way: we are given a family of functions $f_{j} \circ B_{j}$, each one having some degree of symmetry (indeed, these functions are constant on the fibers of the maps $B_{j}$, that are affine subspaces parallel to $\operatorname{ker} B_{j}$ ) and we want to find exponents $p_{j}$ for which the inequality holds with a finite constant $C$ for all choices of functions. Theorem 1.1 gives a complete answer to this question in the Euclidean setting, relating the exponents $p_{j}$ to the geometry of the maps $B_{j}$ and to the scale invariant structure of $\mathbb{R}^{n}$.

An interesting issue is to extend inequality (1.1) to other settings. This problem was already addressed in the works $[9,10]$ where some inequalities were obtained in the case of real spheres and of the permutation group on $d$ elements $S_{d}$ (see also [3, 4] for further results).

In particular in [9, Theorem 1.1] the authors proved that for nonnegative measurable functions $f_{i}$ on the unit sphere $\mathbb{S}^{n-1}$ of $\mathbb{R}^{n}$ depending only on one variable $x_{i}$ (that can be seen as functions $\tilde{f}$ defined on the interval $[-1,1]$ and pulled-back to the sphere by the projection on the $i$-th variable $\left.\pi_{i}: \mathbb{S}^{n-1} \rightarrow[-1,1]\right)$, the estimate

$$
\int_{\mathbb{S}^{n-1}} \prod_{j=1}^{n} \tilde{f}_{j}\left(\pi_{j} x\right) d \sigma \leq \prod_{j=1}^{n}\left\|\tilde{f}_{j} \circ \pi_{j}\right\|_{L^{p}\left(\mathbb{S}^{n-1}\right)}
$$

holds, with $p \geq 2$, where $d \sigma$ is the normalized uniform measure on the sphere. The authors also proved that the inequality is sharp, in the sense that there exist $n$ functions in $L^{p}\left(\mathbb{S}^{n-1}\right)$ for $p<2$, each depending on a different variable, for which the right-hand side of (1.6) is finite and the left-hand side diverges.

Inequality (1.6) can be viewed in two ways:

- it can be compared with Hölder's inequality, but in (1.6) the sum of the reciprocal of the exponents is bigger than one, a condition that cannot be achieved for general functions just by multilinear Hölder's inequality and continuous embeddings of Lebesgue spaces on the sphere, so that, in this sense, inequality (1.6) can be considered an improvement on Hölder's inequality;

- as a Brascamp-Lieb type inequality, plugging in it the estimate $\left\|\tilde{f}_{j} \circ \pi_{j}\right\|_{L^{p}} \lesssim\left\|\tilde{f}_{j}\right\|_{L^{p}([-1,1])}$.

The proof of inequality (1.6) is based on the heat flow method and relies on the fact that the sphere is a compact homogeneous space of Lie groups. Following ideas of [9] in Section 2 of this paper we find inequalities similar to (1.6) in the setting of general compact homogeneous spaces of type $M=K \backslash G$, where $G$ is a connected, unimodular Lie group and $K$ is a closed subgroup of $G$. We endow $M$ with the unique normalized measure $d \mu$ induced by the Haar measure on $G$. We fix a finite set of vector fields $\mathcal{J}$ in the Lie algebra of left 
invariant vector fields $\mathfrak{g}$ of $G$ satisfying Hörmander's bracket generating condition and we construct the sum of squares sub-Laplacian $L$, which is a symmetric, negative, essentially self-adjoint, hypoelliptic operator acting on smooth functions defined on $G$ and on its quotient $M$. By means of the heat semigroup $\left\{e^{t L}\right\}_{t>0}$, we introduce the nonlinear heat flow

$$
v(t, x)=\left(e^{t L} f^{p}\right)^{1 / p},
$$

where $p \geq 1$ and $f \in C^{\infty}(M)$, which is the solution of the nonlinear equation

$$
\partial_{t} v(t, x)=(p-1) \frac{|\nabla v(t, x)|^{2}}{v(t, x)}+L v(t, x),
$$

where $\nabla$ is the gradient with respect to the vector fields in $\mathcal{J}$.

Taking $m$ different nonnegative functions $f_{i} \in C^{\infty}(M)$ and considering their nonlinear evolutions $v_{i}(t, x)$ one can prove that the function

$$
\phi(t)=\int_{M} \prod_{i=1}^{m} v_{i}(t, x) d \mu
$$

is nondecreasing for $p \geq m$. By a comparison between $\lim _{t \rightarrow 0} \phi(t)$ and $\lim _{t \rightarrow \infty} \phi(t)$, it will then follow that

$$
\int_{M} \prod_{i=1}^{m} f_{i} d \mu \leq \prod_{i=1}^{m}\left\|f_{i}\right\|_{L^{p}(M)},
$$

for $p \geq m$. In the case of general functions $f_{i}$ the estimate (1.8) can also be obtained as a straightforward consequence of multilinear Hölder's inequality and continuous embeddings of Lebesgue spaces on $M$. This is not surprising, since with generic functions $f_{i}$ one cannot expect to improve on Hölder's exponents. Nevertheless, if we let the functions involved have some kind of symmetries, we can obtain better exponents not directly deducible by Hölder's inequality and continuous embeddings.

The relevant symmetries in our analysis are those that can be described by means of subsets $\mathcal{A}$ of $\mathcal{J}$ consisting of vector fields that commute with $L$. We call a subset $\mathcal{A}$ of $\mathcal{J}$ maximal if $\langle\mathcal{A}\rangle \cap \mathcal{J}=\mathcal{A}$, where $\langle\mathcal{A}\rangle$ is the smallest Lie subalgebra of $\mathfrak{g}$ containing $\mathcal{A}$. We say that a function $f \in C^{\infty}(M)$ is $\mathcal{A}$-symmetric if $X f=0$ for all vector fields $X$ in $\mathcal{A}$. Functions that are $\mathcal{A}$-symmetric are constant on certain nonintersecting submanifolds that cover the manifold $M$. The commutation property with $L$ of the vector fields in $\mathcal{A}$ ensures that the symmetry is preserved by the heat flow.

The main result of this paper, expressed in Theorem 3.8, says that taking $m$ functions, each $\mathcal{A}_{i}$-symmetric for some maximal subset $\mathcal{A}_{i}$ of $\mathcal{J}$, inequality (1.8) holds for $p$ greater than or equal to a critical $\tilde{p}$ that depends only on the combinatorics of the sets $\mathcal{A}_{i}$ and to prove this we use the fact that for $p \geq \tilde{p}$ the function $\phi$ in (1.7) is nondecreasing. In Theorem 3.10 we obtain an analog of inequality (1.8), but with a different exponent $p_{i}$ for each function $f_{i}$ on the right-hand side. In this case we use the fact that the function

$$
\phi(t)=\int_{M} \prod_{i=1}^{m}\left(e^{t L} f_{i}^{p_{i}}\right)^{1 / p_{i}} d \mu
$$

is nondecreasing if each $p_{i}$ is greater than or equal to a critical $\tilde{p}_{i}$ that again depends on the combinatorics of the sets $\mathcal{A}_{i}$.

As a first application of this machinery, in Section 4 we study the (abelian) case of the torus $\mathbb{T}^{n}=\mathbb{R}^{n} / \mathbb{Z}^{n}$. By means of Theorem 3.8 we are able to recover a result by Calderón in [8] and a family of local geometric Brascamp-Lieb inequalities associated to projections to collections of coordinate variables.

In Section 5 we apply the results of Section 2 to the case of the sphere

$$
\mathbb{S}^{n-1}=S O(n-1) \backslash S O(n)
$$

in $\mathbb{R}^{n}$. We recover the result of [9] for functions depending on one variable and extend it to functions depending on $k$ variables, for $1 \leq k \leq n-1$. 
A function $f$ of $k$ variables can be understood as a function $\tilde{f}$ defined on the $k$-dimensional unit ball $B_{k}$ of $\mathbb{R}^{k}$ and pulled-back to the sphere by the projection $\pi: \mathbb{S}^{n-1} \rightarrow B_{k}$ on the $k$ variables involved.

Let $C(n, k)=\left(\begin{array}{l}n \\ k\end{array}\right)$. We prove that if $f_{1}, \ldots, f_{C(n, k)}$ are nonnegative measurable functions, each depending on a different collection of $k$ variables, denoted with $x_{\omega_{i}}$, where $\omega_{i} \subset\{1, \ldots, n\},\left|\omega_{i}\right|=k$, the inequality

$$
\int_{\mathbb{S}^{n-1}} f_{1}\left(x_{\omega_{1}}\right) \ldots f_{C(n, k)}\left(x_{\left.\omega_{C(n, k)}\right)}\right) d \sigma \leq \prod_{i=1}^{C(n, k)}\left\|f_{i}\right\|_{L^{p}\left(\mathbb{S}^{n-1}\right)}
$$

holds for

$$
p \geq \tilde{p}=\left(\begin{array}{l}
n \\
k
\end{array}\right)-\left(\begin{array}{c}
n-2 \\
k
\end{array}\right) .
$$

Moreover we prove that this inequality is sharp in the sense of [9]. Since for a function $f$ depending on $k$ variables, $\|f\|_{L^{p}}=\|\tilde{f} \circ \pi\|_{L^{p}} \lesssim\|\tilde{f}\|_{L^{p}\left(B_{k}\right)}$, we can interpret (1.10) as a Brascamp-Lieb type inequality.

If we add an additional symmetry to the functions, requiring that each function depends radially on $k$ variables we prove an (again sharp) improvement of inequality (1.10), obtaining a lower critical exponent

$$
\tilde{q}=2\left(\begin{array}{l}
n-2 \\
k-1
\end{array}\right)
$$

This case is studied in Section 6. Inequality (1.10) is first proved for a small range of exponents that is then extended by interpolation. In Section 6 we also study in what range of exponents the inequality can hold, obtaining some optimal result, and provide other examples.

\section{Homogeneous spaces}

For an extensive overview of the setting of homogeneous spaces of Lie groups see [13].

Let $G$ be a connected, unimodular Lie group, with bi-invariant Haar measure $d \mu$ and let $K$ be a closed subgroup so that the homogeneous space $M=K \backslash G$ is compact and has no boundary. Denote by $\pi: G \rightarrow K \backslash G$ the canonical projection. Recall (see [13, Theorem 4.2, Ch. II]) that $M$ is defined as the space of right cosets

$$
M=\{K g: g \in G\}
$$

and has an analytic structure.

If $d \mu$ is the Haar measure on $G$ we have a unique (up to scalars) bi-invariant measure on $K \backslash G$ (cfr. [13, Theorem 1.7, Ch. X]), which we will still denote by $d \mu$, defined as the push-forward of $d \mu$ by means of the projection $\pi$. We assume that this measure is normalized, i.e. $d \mu(M)=1$. We denote with $\tau_{g}$ the left translation on $G$ by the element $g$ and by abuse of notation we will also write $\tau_{g} f$ for $f \circ \tau_{g}$ for functions $f$ on $G$. Recall that a left invariant vector field is a first order differential operator $X$ that commutes with all left translation, i.e. such that

$$
X\left(\tau_{g} f\right)=\left(\tau_{g}\right)(X f)
$$

for all $g \in G$ and $f \in C^{\infty}(G)$. Let $\mathfrak{g}$ be the Lie algebra of the Lie group $G$. As usual, we identify $X \in \mathfrak{g}$ with the corresponding left invariant vector field on $G$ given by the Lie derivative

$$
X f(g)=\left.\frac{d}{d t} f(g \exp (t X))\right|_{t=0},
$$

where exp : $\mathfrak{g} \rightarrow G$ denotes the exponential mapping and $f \in C^{\infty}(G)$.

There is a one-to-one correspondence between smooth real-valued functions $f$ on the quotient space $M=K \backslash G$ and smooth functions $\tilde{f}$ on $G$ that are constant on coset spaces, i.e.

$$
C^{\infty}(K ; G):=\left\{\tilde{f} \in C^{\infty}(G): \tilde{f}(g)=\tilde{f}(k g), \text { for all } k \in K\right\} .
$$


Left invariant differential operators act on smooth functions on $M$ via the pushforward of the map $\pi$ (that we denote with $T \pi$ ). We write $D$ instead of $T \pi(D)$ for differential operators in the universal enveloping algebra $U(\mathfrak{g})$ of $\mathfrak{g}$ acting on $C^{\infty}(M)$. Note that the integration by parts formula

$$
\int_{M}(X f) g d \mu=-\int_{M} f(X g) d \mu
$$

holds on $M$ for $X \in \mathfrak{g}$ and $f, g \in C^{\infty}(M)$. The boundary terms are absent due to the compactness of the quotient.

\subsection{Hörmander systems}

Fix a finite subset $\mathcal{J}=\left\{X_{1}, \ldots, X_{l}\right\}$ of $\mathfrak{g}$.

Definition 2.1. We say that $\mathcal{J}$ is a Hörmander system if $\langle\mathcal{J}\rangle=\mathfrak{g}$, where $\langle\mathcal{J}\rangle$ is the smallest Lie subalgebra containing $\mathcal{J}$.

We now define some differential operators on $M=K \backslash G$ adapted to a Hörmander system J. First of all we define a gradient

$$
\nabla f(x)=\left(X_{1} f(x), \ldots, X_{l} f(x)\right)
$$

for $f \in C^{\infty}(M)$. Next we define a divergence operator

$$
\operatorname{div} F(x)=\sum_{i=1}^{l} X_{i} F_{i}(x)
$$

for $F \in\left(C^{\infty}(M)\right)^{l}$. Finally we define a sum of squares operator, which we will sometimes refer to as subLaplacian,

$$
L f(x)=\sum_{i=1}^{l} X_{i}^{2} f(x)
$$

for $f \in C^{\infty}(M)$. Consider the Hilbert space $L^{2}(M)$ with respect to the measure $d \mu$ with scalar product $\langle\cdot, \cdot\rangle$. The operator $L$ is initially defined in the subspace $C^{\infty}(M)$, which is dense in $L^{2}(M)$ (recall that $M$ is compact). It is immediate to check that the operator $-L$ is symmetric and positive. Since the vector fields in $J$ satisfy Condition 2.1 the operator $L$ is hypoelliptic by Hörmander's theorem. By Nelson's theorem (see [18]) we conclude that the operator $L$ is essentially self-adjoint. Moreover, since $M$ is compact $-L$ has a real discrete nonnegative spectrum $\Sigma \subset \mathbb{R}^{+}$with eigenvalues, counted with multiplicity,

$$
0=\lambda_{0}<\lambda_{1} \leq \cdots \leq \lambda_{k} \leq \ldots,
$$

with $\lambda_{k} \rightarrow \infty$ as $k \rightarrow \infty$. The associated $L^{2}$-normalized eigenfunctions $\varphi_{i}$ form a complete orthonormal system for $L^{2}(M)$. Since the operator $L$ is hypoelliptic, the eigenfunctions are $C^{\infty}(M)$ and in particular they are bounded. Note that $\lambda_{0}$ has multiplicity 1 and $\varphi_{0}=1$.

The spectral theorem provides a functional calculus for the operator $-L$, if $m \in L^{\infty}(\Sigma)$, the operator $m(-L)$ defined by

$$
m(-L) f=\sum_{i=0}^{\infty} m\left(\lambda_{i}\right)\left\langle f, \varphi_{i}\right\rangle \varphi_{i}
$$

is bounded on $L^{2}(M)$. 


\subsection{The heat flow}

We consider the Cauchy problem for the heat equation on $M$ associated to $L$ with initial datum $f$,

$$
\begin{cases}\partial_{t} u(t, x)=L u(t, x) & (t, x) \in \mathbb{R}^{+} \times M \\ u(0, x)=f(x) & x \in M .\end{cases}
$$

It is known (see [22]) that for all $t>0$ the solution at time $t$ of the heat equation with initial datum $f \in C^{\infty}(M)$ is obtained by applying the heat semigroup $e^{t L}$, which is given by the multiplier $e^{-t(\cdot)}: \Sigma \rightarrow \mathbb{R}^{+}$. Explicitly we have

$$
e^{t L} f=\sum_{i=0}^{\infty} e^{-t \lambda_{i}}\left\langle f, \varphi_{i}\right\rangle \varphi_{i}
$$

Fix $p \geq 1$ and consider the evolution for a nonnegative $f \in C^{\infty}(M)$ given by

$$
v(t, x)=\left(e^{t L} f^{p}\right)^{1 / p}(x)
$$

We see that when $p>1, v(t, x)$ is a nonlinear evolution since it satisfies the nonlinear equation

$$
\partial_{t} v(t, x)=(p-1) \frac{|\nabla v(t, x)|^{2}}{v(t, x)}+L v(t, x) .
$$

The solutions to Problem (2.4) and its nonlinear version (2.6) enjoy the following properties.

Proposition 2.2. Let $v: \mathbb{R}^{+} \times M \rightarrow \mathbb{C}$ be a solution of equation (2.6) with $p \geq 1$ and with initial datumf $\in C^{\infty}(M)$. Then the following properties hold.

1. If $f$ is nonnegative, $v(t, x)$ is strictly positive for every $t>0$.

2. The $L^{p}$ mass of the solution is preserved at each time $t>0$ :

$$
\int_{M} v(t, x)^{p} d \mu=\int_{M} f^{p} d \mu .
$$

3. The operators $\left(e^{t L}(\cdot)^{p}\right)^{1 / p}$ enjoy the semigroup property, i.e. $v(t+s, x)=v(s, v(t, x))$.

4. The solution converges to the $L^{p}(M)$ norm of the initial datum at each point $x \in M$, i.e.

$$
\lim _{t \rightarrow \infty} v(t, x)=\left(\int_{M} f^{p} d \mu\right)^{1 / p} .
$$

Proof. Properties (1) and (2) follow from Hunt's theorem (see [14]) for the group $G$ and pass to the quotient $M$ (see [17, Section 2.5]). Property (3) is obvious. Property (4) follows from the fact that 0 is an eigenvalue for $L$ with constant eigenfunction $\varphi_{0}=1$ and that (2.6) converges to $\left(\left\langle f^{p}, \varphi_{0}\right\rangle\right)^{1 / p}=\left(\int_{M} f^{p} d \mu\right)^{1 / p}$ as $t \rightarrow \infty$.

\subsection{A monotonicity result}

Fix $p \geq 1$ and $m \in \mathbb{N}$. Consider a set $\left\{f_{1}, \ldots, f_{m}\right\}$ of nonnegative smooth functions on $M$ and the associated flows

$$
v_{i}(t, x)=\left(e^{t L} f_{i}^{p}\right)^{1 / p}(x)
$$

For fixed $t>0$ consider the function of $t$ given by the integration over $M$ of the product of the nonlinear evolutions $v_{i}(t, x)$ :

$$
\phi(t)=\int_{M} \prod_{i=1}^{m} v_{i}(t, x) d \mu .
$$


Lemma 2.3. If the function (2.9) is nondecreasing, the following inequality holds:

$$
\int_{M} \prod_{i=1}^{m} f_{i} d \mu \leq \prod_{i=1}^{m}\left\|f_{i}\right\|_{L^{p}(M)} .
$$

Proof. Since $\phi(t)$ is nondecreasing we have

$$
\lim _{t \rightarrow 0} \phi(t) \leq \lim _{t \rightarrow \infty} \phi(t)
$$

On the left-hand side we obviously obtain the integral of the product of the initial data. For the right-hand side, by Property (4) of Proposition 2.2, each $v_{i}(t, x)$ converges to $\left\|f_{i}\right\|_{L^{p}(M)}$ and the result follows since $\int_{M} 1 d \mu=$ 1.

Remark 2.4. Since the space $M$ is compact, the best constant in inequality (2.10) is 1 and is attained by constant functions, since for $a \in \mathbb{R}^{+},\|a\|_{L^{p}(M)}=a$ for $1 \leq p \leq \infty$.

Remark 2.5. At first sight the estimate (2.10) looks like Hölder's inequality. Note, however, that in (2.10) there are in general no constraints on the exponent $p$. In particular $\sum_{i=1}^{m} p^{-1}$ need not be 1 . In fact, proving the monotonicity of $\phi$ under certain assumptions that will be made clear later, we will get exponents that do not satisfy Hölder's condition.

We first find an explicit formula for the time derivative of $\phi(t)$. Note that $\phi$ is differentiable in time. Since by Property (1) of Proposition 2.2, each $v_{i}(t, x)$ is strictly positive, we may define, for $t>0$ and $i=1, \ldots, m$,

$$
\tilde{v}_{i}(t, x)=\log \left(v_{i}(t, x)\right)
$$

and

$$
G(t, x)=\prod_{i=1}^{m} v_{i}(t, x)
$$

Proposition 2.6. Under the assumptions above we have

$$
\begin{aligned}
\frac{d}{d t} \phi(t) & =(p-1) \sum_{i=1}^{m} \int_{M} \sum_{j=1}^{l}\left(X_{j} \tilde{v}_{i}(t, x)\right)^{2} G(t, x) d \mu \\
& -\sum_{i=1}^{m} \sum_{j=1}^{l} \sum_{\substack{k=1 \\
k \neq i}}^{m} \int_{M}\left(X_{j} \tilde{v}_{i}(t, x) X_{j} \tilde{v}_{k}(t, x)\right) G(t, x) d \mu .
\end{aligned}
$$

Proof. By the Leibniz rule and (2.7) we have

$$
\begin{aligned}
\frac{d}{d t} \phi(t) & =\sum_{i=1}^{m} \int_{M} \partial_{t} v_{i}(t, x) \prod_{\substack{j=1 \\
j \neq i}}^{m} v_{j}(t, x) d \mu \\
& =\sum_{i=1}^{m} \int_{M}\left((p-1) \frac{\left|\nabla_{L} v_{i}(t, x)\right|^{2}}{v_{i}(t, x)}+L v_{i}(t, x)\right) \prod_{\substack{j=1 \\
j \neq i}}^{m} v_{j}(t, x) d \mu .
\end{aligned}
$$

We split each integral in the sum into two pieces:

$$
\begin{aligned}
\int_{M}(p-1) \frac{\left|\nabla_{L} v_{i}(t, x)\right|^{2}}{v_{i}(t, x)} \prod_{\substack{j=1 \\
j \neq i}}^{m} v_{j}(t, x) d \mu+\int_{M} L v_{i}(t, x) \prod_{\substack{j=1 \\
j \neq i}}^{m} v_{j}(t, x) d \mu \\
=: I_{i}(t)+I I_{i}(t) .
\end{aligned}
$$


For $I_{i}(t)$ we have

$$
I_{i}(t)=(p-1) \int_{M} \frac{\left|\nabla_{L} v_{i}(t, x)\right|^{2}}{v_{i}(t, x)^{2}} G(t, x) d \mu=(p-1) \int_{M} \sum_{j=1}^{l}\left(X_{j} \tilde{v}_{i}(t, x)\right)^{2} G(t, x) d \mu .
$$

For $I I_{i}(t)$, integrating by parts, we obtain:

$$
I I_{i}(t)=-\sum_{j=1}^{l} \int_{M} X_{j} v_{i}(t, x) X_{j}\left(\prod_{\substack{j=1 \\ j \neq i}}^{m} v_{j}(t, x)\right) d \mu
$$

which, using again the Leibniz rule, gives

$$
\begin{aligned}
I I_{i}(t) & =-\sum_{j=1}^{l} \int_{M} X_{j} v_{i}(t, x) \sum_{\substack{k=1 \\
k \neq i}}^{m}\left(X_{j} v_{k}(t, x) \prod_{\substack{k^{\prime}=1 \\
k^{\prime} \neq i, k}}^{m} v_{k^{\prime}}(t, x)\right) d \mu \\
& =-\sum_{j=1}^{l} \sum_{\substack{k=1 \\
k \neq i}}^{m} \frac{X_{j} v_{i}(t, x)}{v_{i}(t, x)} \frac{X_{j} v_{k}(t, x)}{v_{k}(t, x)}\left(\prod_{k^{\prime}=1}^{m} v_{k^{\prime}}(t, x)\right) d \mu \\
& =-\sum_{j=1}^{l} \sum_{\substack{k=1 \\
k \neq i}}^{m} \int_{M} X_{j} \tilde{v}_{i}(t, x) X_{j} \tilde{v}_{k}(t, x) G(t, x) d \mu .
\end{aligned}
$$

Finally, taking the sum in $i$ we obtain the result.

Remark 2.7. By manipulating the sums, the time derivative of $\phi$ can be equivalently written as

$$
\begin{aligned}
\frac{d}{d t} \phi(t) & =(p-1) \sum_{i=1}^{m} \int_{M} \sum_{j=1}^{l}\left(X_{j} \tilde{v}_{i}(t, x)\right)^{2} G(t, x) d \mu \\
& -2 \sum_{i=1}^{m} \sum_{j=1}^{l} \sum_{k=1}^{i-1} \int_{M}\left(X_{j} \tilde{v}_{i}(t, x) X_{j} \tilde{v}_{k}(t, x)\right) G(t, x) d \mu .
\end{aligned}
$$

We observe that this expression contains all possible square type terms $\left(X_{j} \tilde{v}_{i}\right)^{2}$ and all possible double products $2 X_{j} \tilde{v}_{i} X_{j} \tilde{v}_{k}$ for $j=1, \ldots, l$ and $i, k=1, \ldots, m$, with $i<k$. Hence, if there are enough square type terms, i.e. if $p$ is big enough, it is possible to group the terms up in order to get a sum of nonnegative squares of binomials and (possibly) other nonnegative summands.

One could allow each nonnegative $f_{i} \in C^{\infty}(M)$ to evolve with a different nonlinear evolution. Indeed, one could choose a different $p_{i} \geq 1$ for each $f_{i}$ and define

$$
v_{i}(t, x)=\left(e^{t L} f_{i}^{p_{i}}\right)^{1 / p_{i}}(x)
$$

In this regard, we state a simple modification of Proposition 2.6.

Proposition 2.8. In the hypotheses above we have

$$
\begin{aligned}
\frac{d}{d t} \phi(t) & =\sum_{i=1}^{m} \int_{M}\left(p_{i}-1\right) \sum_{j=1}^{l}\left(X_{j} \tilde{v}_{i}(t, x)\right)^{2} G(t, x) d \mu \\
& -\sum_{i=1}^{m} \sum_{j=1}^{l} \sum_{\substack{k=1 \\
k \neq i}}^{m} \int_{M}\left(X_{j} \tilde{v}_{i}(t, x) X_{j} \tilde{v}_{k}(t, x)\right) G(t, x) d \mu .
\end{aligned}
$$


Proof. The proof is the same as for Proposition 2.6, once noticed that each $v_{i}(t, x)$ solves the equation

$$
\partial_{t} v_{i}(t, x)=\left(p_{i}-1\right) \frac{\left|\nabla v_{i}(t, x)\right|^{2}}{v_{i}(t, x)}+L v_{i}(t, x) .
$$

As a simple consequence of Proposition 2.8 one may obtain multilinear Hölder's inequality for a restricted range of exponents. Indeed, taking $p_{i} \geq m$, the time derivative of function (2.9) can be arranged in the form

$$
\begin{aligned}
\frac{d}{d t} \phi(t) & =\sum_{i=1}^{m} \sum_{j=1}^{l} \int_{M}\left(p_{i}-m\right)\left(X_{j} \tilde{v}_{i}(t, x)\right)^{2} G(t, x) d \mu \\
& +\sum_{k<i} \int_{M} \sum_{j=1}^{l}\left(X_{j} \tilde{v}_{i}-X_{j} \tilde{v}_{k}\right)^{2} G(t, x) d \mu \geq 0,
\end{aligned}
$$

since both summands are nonnegative. Hence by the monotonicity of $\phi$ the inequality

$$
\int_{M} \prod_{i=1}^{m} f_{i} d \mu \leq \prod_{i=1}^{m}\left\|f_{i}\right\|_{L^{p_{i}}(M)} .
$$

holds.

We conclude the section with the following definition, which will be useful in what follows.

Definition 2.9. Let $f_{1}, \ldots, f_{m}$ be nonnegative measurable functions and $p_{i} \geq 1$ for $i=1, \ldots, m$. We say that the inequality

$$
\int_{M} \prod_{i=1}^{m} f_{i} d \mu \leq \prod_{i=1}^{m}\left\|f_{i}\right\|_{L^{p_{i}}}
$$

is nontrivial if $\sum_{i=1}^{m} p_{i}^{-1}>1$, i.e. if it does not follow directly from Hölder's inequality and continuous embeddings of Lebesgue spaces.

\section{Inequalities for functions with symmetries}

As the proof of multilinear Hölder's inequality suggests, the choice of the exponent $p$ depends in a combinatorial fashion on the number of vector fields and on the number of functions. Multilinear Hölder's inequality from this point of view represents the worst case, in which one considers all vector fields of the family $J$ applied to all functions. In what follows we will investigate the cases where some of the functions are annihilated by a subset of the vector fields in the family $\mathcal{J}$.

Definition 3.1. Let $\mathcal{A} \subseteq \mathfrak{g}$. We say that a function $f \in C^{\infty}(M)$ is $\mathcal{A}$-symmetric if $X f=0$ for all $X \in \mathcal{A}$. We denote with $C_{\mathcal{A}}^{\infty}(M)$ the space of $\mathcal{A}$-symmetric functions, which is also an algebra with respect to pointwise multiplication.

Remark 3.2. Since if $X f=0$ and $Y f=0$, then also $[X, Y] f=0$, we see that if a function is $\mathcal{A}$-symmetric, then it is also $\langle\mathcal{A}\rangle$-symmetric. In particular, if $\mathcal{A}_{1}$ and $\mathcal{A}_{2}$ are subsets of $\mathfrak{g}$ such that $\left\langle\mathcal{A}_{1}\right\rangle=\left\langle\mathcal{A}_{2}\right\rangle$, we have that $C_{\mathcal{A}_{1}}^{\infty}(M)=C_{\mathcal{A}_{2}}^{\infty}(M)$.

Functions that are $\mathcal{A}$-symmetric enjoy invariance properties on the group $G$, and hence on the manifold $M$.

Definition 3.3. Let $G^{\prime}<G$ be a Lie subgroup of $G$. We say that $f \in C^{\infty}(G)$ is $G^{\prime}$-invariant if $f\left(g g^{\prime}\right)=f(g)$ for all $g^{\prime} \in G^{\prime}$. 
Remark 3.4. With $G^{\prime}<G$ a connected subgroup of $G$ and $\mathfrak{g}^{\prime}$ its Lie algebra, if $f \in C^{\infty}(G)$ we have that $f \in C_{\mathfrak{g}^{\prime}}^{\infty}(G)$ if and only if $f$ is $G^{\prime}$-invariant.

Next we define a class of sets that will be useful to describe the symmetries we are interested in. Since if $X \in \mathfrak{g}$ commutes with $L$ then $e^{t L} X=X e^{t L}$ for all $t>0$, we introduce the following notion.

Definition 3.5. Let $\mathcal{A} \subset \mathfrak{g}$ and $\mathcal{J}$ be a Hörmander system. We say that $\mathcal{A}$ is an J-set if $L$ commutes with all the elements in $\mathcal{A}$, i.e. $[L, X]=L X-X L=0$.

We are interested in functions that are $\mathcal{A}$-symmetric, with $\mathcal{A}$ some $\mathcal{J}$-set. For these functions we have the following proposition.

Proposition 3.6. Let $\mathcal{A} \subseteq \mathfrak{g}$ be an J-set and let $f \in C_{\mathcal{A}}^{\infty}(M)$. For all $1 \leq p<\infty$. Then $e^{t L} f^{p} \in C_{\mathcal{A}}^{\infty}(M)$ for all $t>0$.

Proof. The case $p=1$ is immediate. For the case $p>1$ it suffices to notice that, if $X \in \mathcal{A}$,

$$
X\left(e^{t L} f^{p}\right)^{1 / p}=\frac{1}{p}\left(e^{t L} f^{p}\right)^{\frac{1-p}{p}} X e^{t L} f^{p}=0,
$$

since $\left[X, e^{t L}\right]=0$ and $f^{p} \in C_{\mathcal{A}}^{\infty}(M)$.

Thus the heat flow preserves the symmetry: if $\mathcal{A}$ is an $\mathcal{J}$-set and if the initial datum is $\mathcal{A}$-symmetric, then so is its evolution, either linear or nonlinear, under the heat equation.

Given a subalgebra $\mathcal{A} \subseteq \mathfrak{g}$ we can consider the vector space of functions $C_{\mathcal{A}}^{\infty}(M)$ that are annihilated by all vector fields in $\mathcal{A}$. The Lie algebra $\mathfrak{g}$ has a (nonunique) direct sum decomposition as a vector space

$$
\mathfrak{g}=\mathcal{A} \oplus \mathcal{B},
$$

where $\mathcal{B}$ is a vector subspace of $\mathfrak{g}$.

By Proposition 2.6, for the task of proving the inequalities we are interested in, it suffices to take into account only the action of vector fields in the system $\mathcal{J}$. So we can only consider $\mathcal{J} \cap \mathcal{A}$ or we may as well consider subalgebras generated by subsets of vectors in $\mathcal{J}$. Different subsets of $\mathcal{J}$ could generate the same subalgebra and we will not distinguish them. This leads us to the following definition.

Definition 3.7. Let $\mathcal{A} \subseteq \mathcal{J}$. We say that $\mathcal{A}$ is maximal in $\mathcal{J}$ if for every $\mathcal{A}^{\prime} \subseteq \mathcal{J}$ such that $\langle\mathcal{A}\rangle=\left\langle\mathcal{A}^{\prime}\right\rangle$, we have that $\mathcal{A}^{\prime} \subseteq \mathcal{A}$.

In other words a maximal subset $\mathcal{A}$ of $\mathcal{J}$ contains all possible brackets and linear combinations of its elements that are still in $\mathcal{J}$. For example, if we take the Hörmander system $\left\{e_{1}, \ldots, e_{n}, e_{1}+e_{2}\right\}$ in the abelian Lie algebra $\mathbb{R}^{n}$, where $\left\{e_{1}, \ldots, e_{n}\right\}$ is a basis, we have that $\left\{e_{1}, e_{2}\right\}$ is not maximal, since the set $\left\{e_{1}, e_{2}, e_{1}+\right.$ $\left.e_{2}\right\}$ is such that $\left\langle\left\{e_{1}, e_{2}\right\}\right\rangle=\left\langle\left\{e_{1}, e_{2}, e_{1}+e_{2}\right\}\right\rangle$, but $\left\{e_{1}, e_{2}, e_{1}+e_{2}\right\} \supsetneq\left\{e_{1}, e_{2}\right\}$.

It is clear that if $\mathcal{A} \subseteq \mathcal{J}$, then $\langle\mathcal{A}\rangle \cap \mathcal{J}$ is maximal in $\mathcal{J}$.

Let us introduce a notation. If $A_{1}, \ldots, A_{m}$ are finite sets, for a multi-index $\boldsymbol{j}=\left(j_{1}, \ldots, j_{m}\right) \in\{0,1\}^{m}$ we denote with

$$
\bigcap_{j} A_{i}=\bigcap_{i: j_{i}=1} A_{i}
$$

the intersection of the sets $A_{i}$ such that $j_{i}=1$. Obviously the length of the multi-index, denoted by $|\boldsymbol{j}|$, gives the number of sets we are considering in the intersection.

Theorem 3.8. Let $\mathcal{A}_{1}, \ldots, \mathcal{A}_{m}$ be maximal subsets of $\mathcal{J}$ that are J-sets. Let $f_{i} \in C_{\mathcal{A}_{i}}^{\infty}(M)$ be nonnegative functions, for $i=1, \ldots, m$. The following inequality holds

$$
\int_{M} \prod_{i=1}^{m} f_{i} d \mu \leq \prod_{i=1}^{m}\left\|f_{i}\right\|_{L^{p}(M)}
$$


for $p \geq \tilde{p}$, where $\tilde{p}$ is the number of occurrences of the most recurrent element among the finite sets $\mathcal{A}_{i}^{c}$, i.e.

$$
\tilde{p}=\max _{a \in \cup_{i} \mathcal{A}_{i}^{c}} \max _{j: \cap_{j} \mathcal{A}_{i}^{c} \ni a}|\boldsymbol{j}| .
$$

The exponent $\tilde{p}$ has a combinatorial nature and is related to the way the vector fields $L_{i, j}$ are distributed among the sets $\mathcal{A}_{i}$. In the next sections we will give several examples in which this quantity is easily computable. It is interesting to notice that the exponent $\tilde{p}$ found by this method is always an integer.

Proof. Since the functions $f_{i}$ are $\mathcal{A}_{i}$-symmetric, and the sets $\mathcal{A}_{i}$ are J-sets, by Proposition 3.6 the nonlinear evolutions, defined in (2.8), are also $\mathcal{A}_{i}$-symmetric. By Proposition 2.6 all possible double products of the form $X_{i} \tilde{v}_{j} X_{i} \tilde{v}_{k}$, with $i<k$ and $X_{i} \in \mathcal{A}_{j}^{c} \cap \mathcal{A}_{k}^{c}$, will appear in the time derivative of $\phi$. Recall that $\tilde{p}-1$ depends on how many square type elements are needed to complete the squares. In order to have positive derivative, we will need $\tilde{p}-1$ to be at least as big as the number of occurrences of the most recurrent vector field among the $\mathcal{A}_{i}^{c}$.

The following sufficient condition to obtain a nontrivial inequality in the sense of Definition 2.9 can be easily proved.

Proposition 3.9. Let $\mathcal{A}_{1}, \ldots, \mathcal{A}_{m}$ be maximal subsets of $\mathcal{J}$ that are J-sets. To obtain a nontrivial inequality from the nonlinear heat flow associated to $L$ the condition

$$
\bigcap_{i=1}^{m} \mathcal{A}_{i}^{c}=\emptyset
$$

must be fulfilled.

Proof. By Theorem 3.8 we have a nontrivial inequality if $\tilde{p}<m$ and this happens if no elements of $\mathcal{J}$ appear in all the sets $\mathcal{A}_{i}^{c}$, yielding condition (3.1).

So far we considered the case where all functions evolve under the same flow, i.e. with the same exponent $p \geq 1$. If we consider different exponents $p_{i}$ for different evolutions we have the following result, which can be obtained by the same monotonicity argument as in the proof of Theorem 3.8. We omit the proof, but we just point out that in this case we use formula (2.8) for the time derivative of $\phi$.

Theorem 3.10. Let $\mathcal{A}_{1}, \ldots, \mathcal{A}_{m}$ be maximal subsets of J that are J-sets. Let $f_{i} \in C_{\mathcal{A}_{i}}^{\infty}(M)$ be nonnegative functions, for $i=1, \ldots, m$. The following inequality holds

$$
\int_{M} \prod_{i=1}^{m} f_{i} d \mu \leq \prod_{i=1}^{m}\left\|f_{i}\right\|_{L^{p_{i}(M)}}
$$

for $p_{i} \geq \tilde{p}_{i}$, where $\tilde{p}_{i}$ is the number of occurrences of the most recurrent element of $\mathcal{A}_{i}^{c}$ among the finite sets $\mathcal{A}_{k}^{c}$, i.e.

$$
\tilde{p}_{i}=\max _{a \in \mathcal{A}_{i}^{c}} \max _{\boldsymbol{j}: \cap_{j} \mathcal{A}_{k}^{c} \ni a}|\boldsymbol{j}| .
$$

\section{The abelian case}

As a first example, in this section we analyze the inequalities discussed in the previous section when the Lie group is $\left(\mathbb{R}^{n},+\right)$. We fix an orthonormal basis $\left\{e_{1}, \ldots, e_{n}\right\}$ of $\mathbb{R}^{n}$ and consider the corresponding Cartesian coordinates $\left(x_{1}, \ldots, x_{n}\right)$. We take the quotient by the discrete subgroup $\mathbb{Z}^{n}$,

$$
\mathbb{R}^{n} / \mathbb{Z}^{n}=\mathbb{T}^{n},
$$


where $\mathbb{T}^{n}$ is the $n$-dimensional torus, which can be understood as the cube $[0,1]^{n}$ in $\mathbb{R}^{n}$ with identifications of opposite sides.

The Lie algebra of $\mathbb{R}^{n}$ is generated by the vector fields $X_{i}=\partial_{x_{i}}$ for $i=1, \ldots, n$. Clearly $\left[X_{i}, X_{j}\right]=0$ for all $i, j=1, \ldots, n$. In this setting a Hörmander system of vector fields must necessarily contain a basis for the Lie algebra, since all commutators are trivial. So let

$$
\mathcal{J}=\left\{Y_{1}, \ldots, Y_{l}\right\}
$$

with $l \geq n$ and let $\left\{Y_{1}, \ldots, Y_{n}\right\}$ be a basis for the Lie algebra. In this abelian setting all subsets $\mathcal{A} \subseteq \mathcal{J}$ are J-sets, since every two vectors commute. So we can pick any subset of $\mathcal{J}$ and we have the following proposition.

Proposition 4.1. A subset $\mathcal{A}=\left\{Y_{i_{1}}, \ldots, Y_{i_{s}}\right\}$ of $\mathcal{J}$, with $1 \leq i_{1}<\cdots<i_{s} \leq l$, is maximal if and only if, for all $X \in \mathcal{J} \backslash \mathcal{A}$,

$$
\operatorname{rank}\left(Y_{i_{1}}, \ldots, Y_{i_{s}}, X\right) \neq \operatorname{rank}\left(Y_{i_{1}}, \ldots, Y_{i_{s}}\right) .
$$

Proof. We know that $\langle\mathcal{A}\rangle \cap \mathcal{J}$ is maximal. Since $\mathfrak{g}$ is abelian, vectors in $\langle\mathcal{A}\rangle \cap \mathcal{J}$ that are not in $\mathcal{A}$ are vectors of $\mathcal{J}$ that are linearly dependent from the vectors in $\mathcal{A}$. Condition (4.1) ensures that $\mathcal{A}$ already contains these vectors.

Let us treat the case $l=n$, i.e. when $\mathcal{J}$ is a basis for $\mathfrak{g}$. In this case all subsets of $\mathcal{J}$ are maximal, so we have $2^{n}$ possible maximal subsets to which we can apply Theorem 3.8. If $\mathcal{A}$ is any subset, the vector space sum decomposition $\mathfrak{g}=\langle\mathcal{A}\rangle \oplus\left\langle\mathcal{A}^{c}\right\rangle$ is also a Lie subalgebras decomposition, meaning that $\left[\langle\mathcal{A}\rangle,\left\langle\mathcal{A}^{c}\right\rangle\right]=\{0\}$. All subsets have maximal complement and we can directly consider the complements of the annihilating sets.

Let us discuss the case where $\mathcal{J}=\left\{X_{1}, \ldots, X_{n}\right\}$. Consider a subset $\mathcal{A} \subseteq \mathcal{J}$ given by $\mathcal{A}=\left\{X_{i_{1}}, \ldots, X_{i_{s}}\right\}$ with $s \leq n$. The Lie subalgebra $\langle\mathcal{A}\rangle$ is just the vector subspace of $\mathfrak{g}$ given by $\operatorname{span}\left(X_{i_{1}}, \ldots, X_{i_{s}}\right)$, which corresponds to the Lie subgroup $\tilde{\mathcal{A}}$ given by $\operatorname{span}\left(e_{i_{1}}, \ldots, e_{i_{s}}\right)$. A nonnegative function $f=f\left(x_{1}, \ldots, x_{n}\right)$ on the torus $\mathbb{T}^{n}$ which is $\mathcal{A}$-symmetric is constant on translates by vectors in the Lie subgroup $\langle\mathcal{A}\rangle$, i.e. $f(x+v)=f(x)$, for all $v \in \tilde{\mathcal{A}}$. In other words, the function $f$ does not depend on the variables $x_{i_{1}}, \ldots, x_{i_{s}}$ and we can think of it as a function of the remaining $n-s$ variables. Suppose for simplicity that $i_{j}=j$, for $j=1, \ldots, s$, then $f$ can be identified with a function

$$
F: \mathbb{R}^{n} / \mathbb{R}^{s} \simeq \mathbb{R}^{n-s} \rightarrow \mathbb{R}^{+}
$$

such that $F\left(x_{s+1}, \ldots, x_{n}\right)=f\left(x_{1}, \ldots x_{s}, x_{s+1} \ldots, x_{n}\right)$ for any $s$-tuple $\left(x_{1}, \ldots, x_{s}\right)$. Equivalently we can write

$$
F=f \circ \pi,
$$

where $\pi: \mathbb{T}^{n} \rightarrow \mathbb{T}^{n-s}$ is the linear projection

$$
\pi\left(x_{1}, \ldots, x_{n}\right)=\left(x_{s+1}, \ldots, x_{n}\right) .
$$

We follow the notation of [8], denoting with $\omega$ finite subsets of $\{1, \ldots, n\}$ and with $x_{\omega}$ the set of variables $\left\{x_{i_{1}}, \ldots, x_{i_{|\omega|}}\right\}$, where $\omega=\left\{i_{1}, \ldots, i_{|\omega|}\right\}$. We denote with $f_{\omega}$ a function only depending on $x_{\omega}$. Note that

$$
\int_{\mathbb{T}^{|\omega|}} f_{\omega}\left(x_{\omega}\right) d x_{\omega}=\int_{\mathbb{T}^{n}} f_{\omega}\left(x_{\omega}\right) d x_{1} \ldots d x_{n},
$$

from which we get that

$$
\left\|f_{\omega}\right\|_{L^{p}\left(\mathbb{T}^{|\omega|}\right)}=\left\|f_{\omega}\right\|_{L^{p}\left(\mathbb{T}^{n}\right)}
$$

for all $p \geq 1$.

Let $C(n, k):=\left(\begin{array}{l}n \\ k\end{array}\right)$. We have the following proposition.

Proposition 4.2. Let $\omega_{1}, \ldots, \omega_{C(n, k)}$ be the possible $k$-tuples of elements in $\{1, \ldots, n\}$, and let $f_{\omega_{i}}$ be nonnegative measurable functions only depending on the collection of variables $\omega_{i}$. The inequality

$$
\int_{\mathbb{T}^{n}}^{C(n, k)} \prod_{i=1}^{C} f_{\omega_{i}}\left(x_{\omega_{i}}\right) d x \leq \prod_{i=1}^{C(n, k)}\left\|f_{\omega_{i}}\right\|_{L^{p}\left(\mathbb{T}^{n}\right)}
$$


holds, for

$$
p \geq \tilde{p}=\left(\begin{array}{l}
n-1 \\
k-1
\end{array}\right)
$$

Proof. In the language developed in this section, the sets $\mathcal{A}_{i}^{c}$ are given by $\left\{X_{i_{1}}, \ldots, X_{i_{k}}\right\}$ and they are in correspondence with the collection of variables $x_{\omega_{i}}$, where $\omega_{i}=\left\{i_{1}, \ldots, i_{k}\right\}$. By Theorem 3.8 it suffices to compute the number of occurrences of the most recurrent element among the $\mathcal{A}_{i}^{c}$, or, equivalently, the most recurrent variable $x_{l}$ among the collections $x_{\omega_{i}}$. It is easy to see that in this case every variable $x_{l}$ appears exactly $\left(\begin{array}{l}n-1 \\ k-1\end{array}\right)$ times.

Remark 4.3. Proposition 4.2 is a local version of a result due to A. P. Calderón in [8] (see also the work of $H$. Finner [11] for further results). In the notation above, Calderón proved the inequality

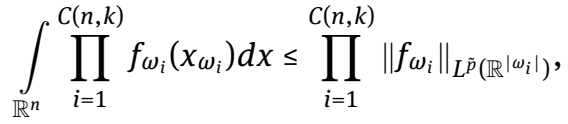

with $\tilde{p}=\left(\begin{array}{l}n-1 \\ k-1\end{array}\right)$, by induction on the cardinality $k$ of the subsets $\omega_{i}$. If the functions $f_{\omega_{i}}$ are supported in the unit cubes of $\mathbb{R}^{\left|\omega_{i}\right|}$, Calderón inequality becomes

$$
\int_{\mathbb{T}^{n}} \prod_{i=1}^{C(n, k)} f_{\omega_{i}}\left(x_{\omega_{i}}\right) d x \leq \prod_{i=1}^{C(n, k)}\left\|f_{\omega_{i}}\right\|_{L^{\tilde{p}}\left(\mathbb{T}^{\mid} \omega_{i} \mid\right)},
$$

which by (4.2) is equivalent to (4.3). The case $k=n-1$ is a local version of Loomis-Whitney inequality (see [16]).

All the estimates above (Calderón inequalities, Loomis-Whitney inequality and their local versions) can be proved by a smart iteration of Hölder's inequality.

Another way of proving this kind of inequalities is the heat flow method used in [5]. Recall that a geometric Brascamp-Lieb inequality is an estimate of the type

$$
\int_{\mathbb{R}^{n}} \prod_{i=1}^{m} f_{i}\left(B_{i} x\right) d x \leq C \prod_{i=1}^{m}\left\|f_{i}\right\|_{L^{p_{i}\left(\mathbb{R}^{\left.n_{i}\right)}\right.},}
$$

where $B_{i}: \mathbb{R}^{n} \rightarrow \mathbb{R}^{n_{i}}$ are surjective linear maps such that $B_{i}^{\star}$ is an isometry, i.e. $B_{i} B_{i}^{\star}=\operatorname{Id}_{\mathbb{R}^{n_{i}}}, f_{i}: \mathbb{R}^{n_{i}} \rightarrow \mathbb{R}^{+}$ are measurable functions, and the relation

$$
\sum_{i=1} p_{i}^{-1} B_{i}^{\star} B_{i}=\operatorname{Id}_{\mathbb{R}^{n}}
$$

is satisfied. In [5] the authors prove that under condition (4.5), inequality (4.4) holds with $C=1$. Restricting the supports of the functions to unit cubes in $\mathbb{R}^{n_{i}}$ this local version of the inequality obviously holds

$$
\int_{\mathbb{T}^{n}} \prod_{i=1}^{m} f_{i}\left(B_{i} x\right) d x \leq \prod_{i=1}^{m}\left\|f_{i}\right\|_{L^{p_{i}\left(\mathbb{T}^{\left.n_{i}\right)}\right.},}
$$

under the same assumption (4.5).

Let us consider the case $m=C(n, k), B_{i}=\pi_{\omega_{i}}$ being the projection onto the set of variables $x_{\omega_{i}}$. The maps $B_{i}^{\star}$ are isometries, being inclusion maps. We have to check for which exponents $p_{i}$ assumption (4.5) is satisfied. It is easy to see that $B_{i}^{\star} B_{i}$ is given by a diagonal matrix such that $\left(B_{i}^{\star} B_{i}\right)_{j j}=1$ if and only if $j \in \omega_{i}$, for $j=1, \ldots, n$. Hence condition (4.5) requires that

$$
\sum_{i: \omega_{i} \ni j} p_{i}^{-1}=1
$$

for $j=1, \ldots, n$. Each sum is made by $\left(\begin{array}{l}n-1 \\ k-1\end{array}\right)$ terms, so that condition (4.5) is certainly satisfied if $p_{i}^{-1}=\left(\begin{array}{l}n-1 \\ k-1\end{array}\right)$ for all $i=1, \ldots, C(n, k)$. We note that the general condition (4.5) gives rise to exponents that are not covered by Proposition 4.2. 


\section{The case of the sphere}

In this section we will find inequalities for functions enjoying some symmetries on spheres. We consider the Euclidean space $\mathbb{R}^{n}$, for $n \geq 2$, with the standard scalar product $\langle\cdot, \cdot\rangle$ and the induced norm $|\cdot|$. Let $\left\{e_{1}, \ldots, e_{n}\right\}$ be an orthonormal basis and $\left(x_{1}, \ldots, x_{n}\right)$ the associated coordinates. The $(n-1)$-dimensional unit sphere is the set

$$
\mathbb{S}^{n-1}=\left\{x \in \mathbb{R}^{n}: x_{1}^{2}+\cdots+x_{n}^{2}=1\right\},
$$

which we endow with the normalized uniform measure $d \sigma$.

The sphere $\mathbb{S}^{n-1}$ can be seen as a homogeneous space of the special orthogonal group

$$
\mathbb{S}^{n-1}=S O(n-1) \backslash S O(n),
$$

where $S O(n-1)$ is thought of as a closed subgroup of $S O(n)$ fixing one direction. The measure $d \sigma$ is, up to normalization, the push-forward through the projection map on the quotient $\mathbb{S}^{n-1}$ of the bi-invariant Haar measure on $S O(n)$.

\subsection{Functions depending on $k$ variables}

In what follows we will use cartesian coordinates to describe points on the sphere. In particular we will often write $f\left(x_{1}, \ldots, x_{n}\right)$ for functions $f: \mathbb{S}^{n-1} \rightarrow \mathbb{R}$, implicitly assuming the condition $x_{1}^{2}+\cdots+x_{n}^{2}=1$.

We will consider functions on the sphere depending on $k$ variables, with $1 \leq k \leq n-1$. Let $\omega=\left\{i_{1}, \ldots, i_{k}\right\}$ be a subset of $\{1, \ldots, n\}$, with $|\omega|=k$ and let $x_{\omega}=\left(x_{i_{1}}, \ldots, x_{i_{k}}\right)$. We will use the notation $\omega^{c}=\left\{i_{k+1}, \ldots, i_{n}\right\}$ for the complement of $\omega$ in $\{1, \ldots, n\}$.

Consider the projection

$$
\pi_{\omega}: \mathbb{S}^{n-1} \rightarrow \mathbb{R}^{k}
$$

that maps $\left(x_{1}, \ldots, x_{n}\right) \mapsto\left(x_{i_{1}}, \ldots, x_{i_{k}}\right)$. The image of the map $\pi_{\omega}$ is the closed unit ball $B_{k}$ in $\mathbb{R}^{k}$.

Definition 5.1. We say that a function $f: \mathbb{S}^{n-1} \rightarrow \mathbb{R}$ depends on $k$ variables, for $1 \leq k \leq n-1$ if there exists a function $\tilde{f}: B_{k} \rightarrow \mathbb{R}$ such that

$$
f=\tilde{f} \circ \pi_{\omega}
$$

for some subset $\omega$ of $\{1, \ldots, n\}$, with $|\omega|=k$.

By abuse of notation we will often write $f\left(x_{\omega}\right)$ for a function on the sphere depending on $k$ variables, meaning $\tilde{f}\left(x_{\omega}\right)$.

Functions on the sphere depending on $k$ variables enjoy special symmetry properties. Indeed, they are constant on $(n-k-1)$-dimensional subspheres of the original sphere. The fiber of a point $y \in B_{k}$ is a sphere $\mathbb{S}^{n-k-1}$ of radius $1-y_{1}^{2}-\cdots-y_{k}^{2}$ in $\mathbb{S}^{n-1}$. Note that for fixed $\omega, \pi_{\omega}^{-1}(y) \neq \pi_{\omega}^{-1}\left(y^{\prime}\right)$ if $y \neq y^{\prime}$, so that the subspheres $\pi_{\omega}^{-1}(y)$ indexed by $y \in B_{k}$ do not intersect each other and cover the whole $\mathbb{S}^{n-1}$.

For convenience of the reader, in the following proposition we recall a well known integration formula for functions depending on $k$ variables (see for example [12, 21]).

Proposition 5.2. Let $\omega=\left\{i_{1}, \ldots, i_{k}\right\}$ be a subset of $\{1, \ldots, n\}$ with $|\omega|=k$, for $1 \leq k \leq n-1$. Let $f: \mathbb{S}^{n-1} \rightarrow \mathbb{R}$ be a function depending on the $k$ variables $x_{\omega}$. The following integration formula holds:

$$
\int_{\mathbb{S}^{n-1}} f\left(x_{\omega}\right) d \sigma=c_{n, k} \int_{B_{k}} f\left(x_{\omega}\right)\left(1-x_{i_{1}}^{2}-\cdots-x_{i_{k}}^{2}\right)^{\frac{n-k-2}{2}} d x_{\omega} .
$$

The constant $c_{n, k}$ depends only on the dimension $n$ and on the number of variables $k$. 
Remark 5.3. Let $\omega$ be as above, with $|\omega|=k$, for $1 \leq k \leq n-2$, and let $f: \mathbb{S}^{n-1} \rightarrow \mathbb{R}$ be a function depending on $k$ variables. Since $\left(1-x_{i_{1}}^{2}-\cdots-x_{i_{k}}^{2}\right) \leq 1$, we have the trivial inequality

$$
\int_{\mathbb{S}^{n-1}} f\left(x_{\omega}\right) d \sigma \lesssim \int_{B^{k}} f\left(x_{\omega}\right) d x_{\omega} .
$$

In this way, we obtain a family of continuous immersions

$$
L^{p}\left(B^{k}, d x_{\omega}\right) \hookrightarrow L^{p}\left(\mathbb{S}^{n-1}, d \sigma\right),
$$

for $1 \leq p \leq \infty$.

\subsection{The Lie algebra of the special orthogonal group}

A basis for $\mathfrak{s o}(n)$, the Lie algebra of $S O(n)$, is given by the vector fields

$$
L_{i, j}=x_{i} \partial_{x_{j}}-x_{j} \partial_{x_{i}},
$$

for $1 \leq i<j \leq n$. Since $L_{j, i}=-L_{i, j}$, the dimension of $\mathfrak{s o}(n)$ is $n(n-1) / 2$.

Let $\delta_{i, j}$ be the Kronecker delta. The bracket of two basis elements $L_{i, j}$ and $L_{k, l}$ is given by

$$
\left[L_{i, j}, L_{k, l}\right]=L_{l, j} \delta_{i, k}+L_{i, l} \delta_{j, k}+L_{j, k} \delta_{i, l}+L_{k, i} \delta_{l, k}
$$

It follows that the commutator of two elements of the basis $\left\{L_{i, j}\right\}_{i<j}$, if not trivial, is again an element of the basis.

This basis will be our Hörmander system J. The corresponding sub-Laplacian is given by

$$
L=\sum_{i<j} L_{i, j}^{2} .
$$

The operator $L$ commutes with all the vector fields $L_{i, j}$, since it is the quadratic Casimir operator, which is an element of the center of the universal enveloping algebra $U(\mathfrak{s o}(n))$. Note also that $L$ is the Laplace-Beltrami operator on $S O(n)$ with the Riemannian metric induced by the Killing form (see [13]).

\subsection{Structure of maximal subsets}

We now discuss the structure of maximal subsets of $\mathcal{J}=\left\{L_{i, j}\right\}_{i<j}$. In order to visualize the subsets of $\mathcal{J}$ we associate to the vector field $L_{i, j}$ the pair $(i, j)$. Given a subset $\mathcal{A} \subseteq\{(i, j)\}_{i<j}$, we can relate to it an undirected simple graph $G_{\mathcal{A}}=(V, E)$ where the set of vertices $V$ is given by $\{1, \ldots, n\}$ and the edges $E$ are given by the (unordered) pairs $(i, j) \in \mathcal{A}$, so that we identify $\mathcal{A}$ with $E$.

The following proposition holds.

Proposition 5.4. Let $\mathcal{A}$ be a subset of $\mathcal{J}$. $\mathcal{A}$ is a maximal subset if and only if the associated graph $G_{\mathcal{A}}=(V, E)$ splits in complete connected components.

Proof. Note that if $(a, b) \in E$ and $(b, c) \in E$, then $(a, c) \in E$ by the maximality assumption on $\mathcal{A}$ and (5.3). Since connected components are path connected, each connected component of a graph associated to a maximal subset is complete. The converse is straightforward, again by (5.3).

We also have the following result.

Proposition 5.5. Let $\mathcal{A}$ be a maximal subset of $\mathcal{J}$ and $G_{\mathcal{A}}=(V, E)$ its associated graph. Each complete connected component $\widetilde{G}=(\widetilde{V}, \widetilde{E})$, identifying $\widetilde{E}$ with a subset of $\mathcal{J}$, has the property that $\langle\widetilde{E}\rangle \simeq \mathfrak{s o}(|\widetilde{V}|)$. 
Proof. Let $\widetilde{V}=\left\{i_{1}, \ldots, i_{k}\right\}$, with $i_{1}<\cdots<i_{k}$, so that $|\widetilde{V}|=k$. Since $\widetilde{G}$ is complete, $\widetilde{E}$ contains all the edges in $E$ with vertices in $\widetilde{V}$. It is easy to see that, by property (5.3), the map $\langle\widetilde{E}\rangle \rightarrow \mathfrak{s o}(k)$ that maps $L_{i_{j}, i_{l}} \mapsto L_{j, l}$, for $i_{j}<i_{l}$, is a Lie algebra isomorphism. Moreover the set $\widetilde{E}$ is a basis for $\langle\widetilde{E}\rangle$.

Let us introduce some notation. Let $\alpha=\left(\alpha^{1}, \ldots, \alpha^{n}\right) \in\{0,1\}^{n}$ be a multi-index and denote by $|\alpha|=$ $\alpha^{1}+\cdots+\alpha^{n}$ its length. The scalar product $\alpha \cdot \beta=\alpha^{1} \beta^{1}+\cdots+\alpha^{n} \beta^{n}$ indicates the number of 1 's in common between $\alpha$ and $\beta$, so that two multi-indices are orthogonal if they do not have 1's in common.

We will denote with $\mathfrak{s o}_{\alpha}$ the Lie algebra isomorphic to $\mathfrak{s o}(|\alpha|)$ generated by the set $\left\{L_{k, l}: \alpha^{k}=\alpha^{l}=1\right\}$. We can deduce the following theorem describing the structure of subalgebras generated by maximal subsets associated to basis systems of $\mathfrak{s o}(n)$.

Theorem 5.6. Let $\mathcal{A}$ be a maximal subset of $\mathcal{J}=\left\{L_{i, j}\right\}_{i<j}$. Then there exist multi-indices $\alpha_{1}, \ldots, \alpha_{N}$ pairwise orthogonal, with $\left|\alpha_{1}\right| \geq\left|\alpha_{2}\right| \geq \cdots \geq\left|\alpha_{N}\right|$ and $\left|\alpha_{1}\right|+\cdots+\left|\alpha_{N}\right| \leq n$, such that

$$
\langle\mathcal{A}\rangle=\bigoplus_{k=1}^{N} \mathfrak{s o}_{\alpha_{k}},
$$

where on the right-hand side we have a direct sum of Lie algebras, i.e. each subalgebra commutes with the others.

Proof. By Proposition 5.4 and Proposition 5.5 the graph associated to $\mathcal{A}$ splits in $N$, say, complete connected components $G_{\alpha_{i}}=\left(V_{\alpha_{i}}, E_{\alpha_{i}}\right)$, where $i=1, \ldots, N$, each component describing a graph associated to a basis system of a Lie algebra of type $\mathfrak{s o}(k)$ for some $k$. Without loss of generality we can assume that $\left|V_{\alpha_{1}}\right| \geq \cdots \geq$ $\left|V_{\alpha_{N}}\right|$ so that $\left|\alpha_{1}\right| \geq \cdots \geq\left|\alpha_{N}\right|$. The multi-indices are pairwise orthogonal since the graphs $G_{\alpha_{i}}$ are disconnected so that $V_{\alpha_{i}} \cap V_{\alpha_{j}}=\emptyset$ for $i \neq j$. It is clear that $\left|\alpha_{1}\right|+\cdots+\left|\alpha_{N}\right|=|V| \leq n$. Finally, since the multi-indices are pairwise orthogonal, in view of formula (5.3), fixing $k \neq l, 1 \leq k, l \leq N$, we have that $\left[L_{i_{1}, j_{1}}, L_{i_{2}, j_{2}}\right]=0$ for all $i_{1}, j_{1}$ such that $\alpha_{k}^{i_{1}}=\alpha_{k}^{j_{1}}=1$ and $i_{2}, j_{2}$ such that $\alpha_{l}^{i_{2}}=\alpha_{l}^{j_{2}}=1$, and by linearity the same holds for all brackets between elements of $\mathfrak{s o}_{\alpha_{l}}$ and $\mathfrak{s o}_{\alpha_{k}}$. Thus the sum in (5.5) is direct.

We now study the properties of functions annihilated by maximal subsets of vectors in J. First we consider the case of a singleton, i.e. $\mathcal{A}=\left\{L_{i, j}\right\}$.

Lemma 5.7. Let $f: \mathbb{S}^{n-1} \rightarrow \mathbb{R}$, with $f \in C^{\infty}\left(\mathbb{S}^{n-1}\right)$ and $L_{i, j}$ be as above. Then $L_{i, j} f(x)=0$ for all $x \in \mathbb{S}^{n-1}$ if and only if there exists a function $\tilde{f}$ such that

$$
f\left(x_{1}, \ldots, x_{i}, \ldots, x_{j}, \ldots, x_{n}\right)=\tilde{f}\left(x_{i}^{2}+x_{j}^{2}, x_{1}, \ldots, \hat{x}_{i}, \ldots, \hat{x}_{j}, \ldots, x_{n}\right),
$$

for all $\left(x_{1}, \ldots, x_{n}\right) \in \mathbb{S}^{n-1}$, where by $\hat{x}_{i}$ we mean that the variable $x_{i}$ is not appearing.

Proof. Clearly, (5.6) implies

$$
L_{i, j} f\left(x_{1}, \ldots, x_{n}\right)=L_{i, j} \tilde{f}\left(x_{i}^{2}+x_{j}^{2}, x_{1}, \ldots, x_{n}\right)=2 x_{i} x_{j} D_{1} \tilde{f}-2 x_{j} x_{i} D_{1} \tilde{f}=0
$$

for all $x \in \mathbb{S}^{n-1}$, where $D_{1}$ denotes the partial derivative with respect to the first variable of $\tilde{f}$. Conversely, suppose that $f$ satisfies $L_{i, j} f(x)=0$ for all $x \in \mathbb{S}^{n-1}$. Since $L_{i, j}$ is the infinitesimal generator of rotations in the $x_{i} x_{j}$-plane, it fixes circles of the type $x_{i}^{2}+x_{j}^{2}=r^{2} ; f$, being annihilated by $L_{i, j}$, is constant on these circles, thus it depends on $x_{i}$ and $x_{j}$ through $x_{i}^{2}+x_{j}^{2}$.

An analogous property holds if we consider functions annihilated by a maximal subset $\mathcal{A}$ of $\mathcal{J}$ whose generated Lie algebra is isomorphic to $\mathfrak{s o}(k)$.

Lemma 5.8. Let $f: \mathbb{S}^{n-1} \rightarrow \mathbb{R}$, with $f \in C^{\infty}\left(\mathbb{S}^{n-1}\right)$ and $\mathcal{A}$ be a maximal subset of J such that $\langle\mathcal{A}\rangle \simeq \mathfrak{s o}(k)$ for some $k \leq n$, i.e. $\mathcal{A}=\left\{L_{i_{j}, i_{l}}\right\}_{i_{j}<i_{l}}$ with $i_{j}, i_{l} \in\left\{i_{1}, \ldots, i_{k}\right\} \subseteq\{1, \ldots, n\}$. Then $L_{i_{j}, i_{l}} f(x)=0$ for all $x \in \mathbb{S}^{n-1}$ and $L_{i_{j}, i_{l}} \in \mathcal{A}$ if and only if there exists a function $\tilde{f}$ such that

$$
f\left(x_{1}, \ldots, x_{i_{1}}, \ldots, x_{i_{k}}, \ldots, x_{n}\right)=\tilde{f}\left(x_{i_{1}}^{2}+\cdots+x_{i_{k}}^{2}, x_{1}, \ldots, \hat{x}_{i_{1}}, \ldots, \hat{x}_{i_{k}}, \ldots, x_{n}\right),
$$

for all $\left(x_{1}, \ldots, x_{n}\right) \in \mathbb{S}^{n-1}$. 
Proof. The assertion is proved arguing as in Lemma 5.7, once noted that the subalgebra $\langle A\rangle$ generates the rotations in the $k$-plane related to the coordinates $x_{i_{1}}, \ldots, x_{i_{k}}$.

By abuse of notation we will just write $f$ in place of $\tilde{f}$.

Remark 5.9. If a function $f \in C^{\infty}\left(\mathbb{S}^{n-1}\right)$ is $\mathcal{A}$-symmetric with respect to a maximal subset $\mathcal{A}$ of $\mathcal{J}$ such that $\langle\mathcal{A}\rangle \simeq \mathfrak{s o}_{\alpha}$ for some multi-index $\alpha$ with $|\alpha|=k$, the function $f$ is a function of $n-k$ variables in the sense of Definition (5.1). Without loss of generality, assume $\alpha^{i}=1$ for $i=1, \ldots, k$ and zero otherwise. By Lemma 5.8 we have

$$
f\left(x_{1}, \ldots, x_{n}\right)=f\left(x_{1}^{2}+\cdots+x_{k}^{2}, x_{k+1}, \ldots, x_{n}\right)=f\left(1-x_{k+1}^{2}-\cdots-x_{n}^{2}, x_{k+1}, \ldots, x_{n}\right),
$$

so that $f$ is a function of the $n-k$ variables $x_{k+1}, \ldots, x_{n}$.

A generic maximal subset $\mathcal{A}$ of $\mathcal{J}$ splits by Theorem 5.6 into $N$ disjoint subsets, labeled by a family of multi-indices $\alpha_{i}$. Each of these subsets generates a subalgebra of $\mathfrak{s o}(n)$ isomorphic to $\mathfrak{s o}\left(\left|\alpha_{i}\right|\right)$. In Theorem 5.6 we ordered these subsets by cardinality. We will interpret the splitting in the following way: the subalgebra related to the multi-index $\alpha_{1}$ tells us on how many variables the functions annihilated by $\mathcal{A}$ depend, as explained in Remark 5.9; the subalgebras related to the multi-indices $\alpha_{i}$, for $2 \leq i \leq N$ give instead information concerning radiality in the variables. To be more precise, functions in $C^{\infty}\left(\mathbb{S}^{n-1}\right)$ that are $\mathcal{A}$-symmetric depend on the $n-\left|\alpha_{1}\right|$ variables $x_{i}$ for which $\alpha_{1}^{i}=0$, and depend radially on the collections of $\left|\alpha_{i}\right|$ variables associated to each multi-index $\alpha_{i}$ (that are disjoint by the orthogonality of the multi-indices).

Example 5.10. Consider the maximal system in $\mathfrak{s o}(7)$ given by $\mathcal{A}=\left\{L_{5,6}, L_{5,7}, L_{6,7}, L_{1,2}, L_{3,4}\right\}$, that splits as $\left\{L_{5,6}, L_{5,7}, L_{6,7}\right\} \sqcup\left\{L_{1,2}\right\} \sqcup\left\{L_{3,4}\right\}$, and whose generated subalgebra $\langle\mathcal{A}\rangle$ splits therefore as

$$
\mathfrak{s o}_{\alpha_{1}}(3) \oplus \mathfrak{s o}_{\alpha_{2}}(2) \oplus \mathfrak{s o}_{\alpha_{3}}(2),
$$

with $\alpha_{1}=(0,0,0,0,1,1,1), \alpha_{2}=(1,1,0,0,0,0,0), \alpha_{3}=(0,0,1,1,0,0,0)$. A function $f \in C^{\infty}\left(\mathbb{S}^{n-1}\right)$ that is $\mathcal{A}$-symmetric will depend on the $n-\left|\alpha_{1}\right|=7-3=4$ variables $x_{1}, x_{2}, x_{3}, x_{4}$ and will be radial in the collections of variables $x_{1}, x_{2}$ associated to $\alpha_{2}$ and $x_{3}, x_{4}$ associated to $\alpha_{3}$. So it will be written as

$$
f\left(x_{1}^{2}+x_{2}^{2}, x_{3}^{2}+x_{4}^{2}\right) \text {. }
$$

Remark 5.11. We stick to the convention of ordering the subsets by cardinality. We remark that all orderings are equivalent. Indeed, in Example 5.10 one could have considered instead the splitting

$$
\mathfrak{s o} \alpha_{2}(2) \oplus \mathfrak{s o} \alpha_{1}(3) \oplus \mathfrak{s o} \alpha_{3}(2) .
$$

In this point of view, a function $f \in C^{\infty}\left(\mathbb{S}^{n-1}\right)$ that is $\mathcal{A}$-symmetric is a function of the $n-\left|\alpha_{2}\right|=7-2=5$ variables $x_{3}, x_{4}, x_{5}, x_{6}, x_{7}$, radial in the collections of variables $x_{5}, x_{6}, x_{7}$ associated to $\alpha_{1}$ and $x_{3}, x_{4}$ associated to $\alpha_{3}$. So it can be written as

$$
f\left(x_{3}^{2}+x_{4}^{2}, x_{5}^{2}+x_{6}^{2}+x_{7}^{2}\right),
$$

but since $x_{5}^{2}+x_{6}^{2}+x_{7}^{2}=1-x_{1}^{2}-x_{2}^{2}-x_{3}^{2}-x_{4}^{2}$, we can reduce the dependence to $x_{3}^{2}+x_{4}^{2}$ and $x_{1}^{2}+x_{2}^{2}$, thus obtaining the same numerology as in Example 5.10.

In the rest of the section we will study some interesting instances of multilinear inequalities of the type (2.10) related to the system $\mathcal{J}=\left\{L_{i, j}\right\}_{i<j}$ described above. We will obtain nontrivial inequalities in the sense of Definition 2.9. As we saw, functions involved in the inequalities have symmetry properties determined by the maximal system $\mathcal{A}$ that annihilates them. We will also be able to show for some of the inequalities that the exponents $\tilde{p}$ found by means of Theorem 3.8 are sharp in a sense that we make precise with the following definition.

Definition 5.12. We will say that the exponent $\tilde{p}$ is sharp if the inequality

$$
\int_{\mathbb{S}^{n-1}} \prod_{i=1}^{m} f_{i} d \mu \leq \prod_{i=1}^{m}\left\|f_{i}\right\|_{L^{p}\left(\mathbb{S}^{n-1}\right)},
$$


holds for $p=\tilde{p}$ and is false for $p<\tilde{p}$, i.e. there exist functions $f_{i}$ for which the right-hand side is finite and the left-hand side diverges.

\subsection{Inequalities for functions depending on $\boldsymbol{k}$ variables}

The first inequality we discuss was discovered by Carlen, Lieb and Loss in [9] and it is an inequality for $n$ functions on the sphere $\mathbb{S}^{n-1}$ each depending on a single different variable. The inequality is the following.

Theorem 5.13 ([9]). Let $f_{1}, \ldots, f_{n}$ be nonnegative measurable functions, $f_{i}:[-1,1] \rightarrow \mathbb{R}^{+}$. The inequality

$$
\int_{\mathbb{S}^{n-1}} f_{1}\left(x_{1}\right) \ldots f_{n}\left(x_{n}\right) d \sigma \leq \prod_{i=1}^{n}\left\|f_{i}\right\|_{L^{p}\left(\mathbb{S}^{n-1}\right)}
$$

holds for $p \geq \tilde{p}=2$. Moreover inequality (5.8) is sharp in the sense of Definition 5.12.

We will now generalize this result of [9] to functions depending on $1 \leq k \leq n-1$ variables and thus obtain Theorem 5.13 as a corollary. The proof for this general case is based on Theorem 3.8, which is in the spirit of the original proof of [9]. We will also give a proof of the sharpness by producing an explicit counterexample.

The case of functions depending on $n-1$ variables is the easiest one and we treat it separately. In this case we have $\left(\begin{array}{c}n \\ n-1\end{array}\right)=n$ possible $(n-1)$-tuples of variables, which correspond to empty maximal systems $\mathcal{A}_{i}$, for which $\left\langle\mathcal{A}_{i}\right\rangle=\{0\}$. Indeed, functions depending on $n-1$ variables are almost generic functions, as explained at the beginning of the section, and there is no hope to obtain something better than Hölder's inequality, i.e. $\tilde{p}=n$. This is confirmed by Theorem 3.8, since each element in each $\mathcal{A}_{i}^{c}=\mathcal{J}$ occurs in all $\mathcal{A}_{k}^{c}$, for $k=1, \ldots, n$.

Let us now consider the case of functions depending on $1 \leq k \leq n-2$ variables. We have $\left(\begin{array}{l}n \\ k\end{array}\right):=C(n, k)$ possible choices ok $k$-tuples out of the set $\{1, \ldots, n\}$. We will label them as $\omega_{1}, \ldots, \omega_{C(n, k)}$ following the notation introduced in Section 4. To each collection of variables $\omega_{i}=\left\{i_{1}, \ldots, i_{k}\right\}$ corresponds a maximal subset $\mathcal{A}_{i}$ which contains the vector fields $L_{j, l}$ for which $j, l \neq i_{s}$ for all $s=1, \ldots, k$.

The subalgebra generated by each maximal subset $\mathcal{A}_{i}$ is isomorphic to $\mathfrak{s o}(n-k)$ and the splitting of $\left\langle\mathcal{A}_{i}\right\rangle$ given by Theorem 5.6 has just one direct summand $\mathfrak{s o}_{\alpha_{i}}$, with $\alpha_{i}$ a multi-index such that $\alpha_{i}^{j}=0$ if $j \in \omega_{i}$, for $j=1, \ldots, n$. By Remark 5.9 a function $f \in C^{\infty}\left(\mathbb{S}^{n-1}\right)$ that is $\mathcal{A}_{i}$-symmetric is a function of the variable $x_{\omega_{i}}$ in $\mathbb{R}^{k}$. As we saw we can think of a function depending on $x_{\omega_{i}}$ as a function defined on the $k$-dimensional unit ball $B_{k} \subset \mathbb{R}^{k}$, pulled back to the sphere $\mathbb{S}^{n-1}$ via the projection $\pi_{\omega_{i}}: \mathbb{S}^{n-1} \rightarrow B_{k}$, mapping a point $x \in \mathbb{S}^{n-1}$ to $x_{\omega_{i}}$. We will write $f\left(x_{\omega_{i}}\right)$ for $f\left(\pi_{\omega_{i}}(x)\right)$, with $x \in \mathbb{S}^{n-1}$. We have the following theorem.

Theorem 5.14. Let $f_{1}, \ldots, f_{C(n, k)}$ be nonnegative measurable functions, $f_{i}: B_{k} \rightarrow \mathbb{R}^{+}$. The inequality

$$
\int_{\mathbb{S}^{n-1}} f_{1}\left(x_{\omega_{1}}\right) \ldots f_{C(n, k)}\left(x_{\left.\omega_{C(n, k)}\right)} d \sigma \leq \prod_{i=1}^{C(n, k)}\left\|f_{i}\right\|_{L^{p}\left(\mathbb{S}^{n-1}\right)}\right.
$$

holds for

$$
p \geq \tilde{p}=\left(\begin{array}{l}
n \\
k
\end{array}\right)-\left(\begin{array}{c}
n-2 \\
k
\end{array}\right) .
$$

Moreover inequality (5.9) is sharp in the sense of Definition 5.12.

Remark 5.15. For $k=1$ we recover the result of [9]. Note that inequality (5.9) is nontrivial in the sense of Definition 2.9 for $n \geq 3$, since $\tilde{p}<C(n, k)$.

Proof. By Theorem 3.8, the exponent $\tilde{p}$ is given by the number of occurrences of the most recurrent element among the sets $\mathcal{A}_{i}^{c}$, for $i=1, \ldots, C(n, k)$. As we said, the elements of $\mathcal{A}_{i}^{c}$ are vector fields of type $L_{j, l}$ with either $j$ or $l$ or both $j, l$ in $\omega_{i}$. So an element $L_{j, l}$ will occur in all $\mathcal{A}_{i}^{c}$ apart from those for which $j, l \notin \omega_{i}$. The 
number of sets $\omega_{i}$ made of $k$ elements taken from $\{1, \ldots, n\}$ that do not contain two fixed elements $j, l$ is $\left(\begin{array}{c}n-2 \\ k\end{array}\right)$. This means that each vector field will occur in exactly

$$
\tilde{p}=\left(\begin{array}{l}
n \\
k
\end{array}\right)-\left(\begin{array}{c}
n-2 \\
k
\end{array}\right)
$$

sets $\mathcal{A}_{i}^{c}$, proving the first half of the theorem.

To show that $\tilde{p}$ is sharp we construct a counterexample. We consider functions $f_{i}: B_{k} \rightarrow \mathbb{R}^{+}$, where $B_{k}$ is the unit ball in $\mathbb{R}^{k}$, of the form

$$
f_{i}\left(x_{\omega_{i}}\right)=\left(\left|x_{i_{1}}\right|\left|x_{i_{2}}\right| \ldots\left|x_{i_{k}}\right|\right)^{-\gamma / k}+\left(1-x_{i_{1}}^{2}\right)^{-\gamma \delta / 2}+\cdots+\left(1-x_{i_{k}}^{2}\right)^{-\gamma \delta / 2},
$$

where $\gamma, \delta$ are positive constants to be determined. We remark that for $k=1$ this set of functions reduces to the counterexample for Theorem 5.13 contained in [9]. The right-hand side of inequality (5.9) must be finite. We first compute the $L^{p}$ norm of these functions. Without loss of generality we focus on the case $\omega=\{1,2, \ldots, k\}$ and work with $f\left(x_{1}, \ldots, x_{k}\right)$. Let $p \geq 1$. We have

$$
\begin{aligned}
\|f\|_{L^{p}\left(\mathbb{S}^{n-1}\right)}^{p} & \lesssim \int_{\mathbb{S}^{n-1}}\left[\left(\left|x_{1}\right|\left|x_{2}\right| \ldots\left|x_{k}\right|\right)^{-\gamma p / k}+\left(1-x_{1}^{2}\right)^{-\gamma \delta p / 2}+\cdots+\left(1-x_{k}^{2}\right)^{-\gamma \delta p / 2}\right] d \sigma \\
& =\int_{\mathbb{S}^{n-1}}\left(\left|x_{1}\right|\left|x_{2}\right| \ldots\left|x_{k}\right|\right)^{-\gamma p / k} d \sigma+\sum_{i=1}^{k} \int_{\mathbb{S}^{n-1}}\left(1-x_{i}^{2}\right)^{-\gamma \delta p / 2} d \sigma \\
& =: I_{0}+\sum_{i=1}^{k} I_{i} .
\end{aligned}
$$

For the first term $I_{0}$ we have

$$
I_{0} \lesssim \int_{B_{k}}\left(\left|x_{1}\right| \ldots\left|x_{k}\right|\right)^{-\gamma p / k}\left(1-x_{1}^{2}-\cdots-x_{k}^{2}\right)^{(n-k-2) / 2} d x_{1} \ldots d x_{k} \leq \prod_{i=1}^{k} \int_{-1}^{1}\left|x_{i}\right|^{-\gamma p / k} d x_{i}
$$

where we used the integration formula (5.1), the fact that $\left(1-x_{1}^{2}-\cdots-x_{k}^{2}\right)^{(n-k-2) / 2} \leq 1$ in $B_{k}$, since $k \leq n-2$, and also that $B_{k} \subset[-1,1]^{k}$. So $I_{0}$ is finite if $\gamma p<k$. For each of the terms $I_{i}$ we have

$$
I_{i}=\int_{-1}^{1}\left(1-x_{i}^{2}\right)^{-\gamma \delta p / 2}\left(1-x_{i}^{2}\right)^{(n-3) / 2} d x_{i}
$$

by (5.1). So $I_{i}$ is finite whenever $\gamma \delta p<(n-1)$. We conclude that the right-hand side of (5.9) is finite if

$$
\gamma p<\min \left\{k, \frac{n-1}{\delta}\right\}
$$

To estimate the left-hand side of (5.9) we pass to polar coordinates in the hyperplane $\mathbb{R}^{n-1}$ with coordinates $x_{1}, \ldots, x_{n-1}$; on the sphere $\mathbb{S}^{n-1}$ the variable $\left|x_{n}\right|$ will then be $\left(1-\rho^{2}\right)^{1 / 2}$. There are $\left(\begin{array}{c}n-1 \\ k\end{array}\right)$ functions not involving the $x_{n}$ variable, and $\left(\begin{array}{l}n-1 \\ k-1\end{array}\right)$ involving it. For the functions not depending on $x_{n}$ we select the first summand of (5.10), for those depending on $x_{n}$ we select the summand $\left(1-x_{n}^{2}\right)^{-\gamma \delta / 2}$.

So for the left-hand side we have:

$$
\begin{aligned}
& \int_{\mathbb{S}^{n-1}}^{C(n, k)} \prod_{i=1}^{1} f_{i}\left(x_{\omega_{i}}\right) d \sigma \geq \int_{\mathbb{S}^{n-1}}\left[\prod_{\omega_{i} \ngtr x_{n}}\left|x_{i_{1}}\right| \ldots\left|x_{i_{k}}\right|\right]^{-\frac{\gamma}{k}}\left(1-x_{n}^{2}\right)^{-\frac{\gamma \delta}{2}\left(\begin{array}{c}
n-1 \\
k-1
\end{array}\right)} d \sigma \\
& \geq \int_{0}^{1}\left(\rho^{k}\right)^{-\frac{\gamma}{k}\left(\begin{array}{c}
n-1 \\
k
\end{array}\right)}\left(\rho^{2}\right)^{-\frac{\gamma \delta}{2}\left(\begin{array}{c}
n-1 \\
k-1
\end{array}\right)} \rho^{n-2} d \sigma=\int_{0}^{1} \rho^{-\left(\gamma\left(\begin{array}{c}
n-1 \\
k
\end{array}\right)+\gamma \delta\left(\begin{array}{c}
n-1 \\
k-1
\end{array}\right)\right)+n-2} \frac{d \rho}{\sqrt{1-\rho^{2}}},
\end{aligned}
$$


where we used the trivial fact that $\left|x_{i}\right| \leq\left(x_{1}^{2}+\cdots+x_{n-1}^{2}\right)^{1 / 2}$, for $i=1, \ldots, n-1$.

The left-hand side of (5.9) diverges when $-\left[\gamma\left(\begin{array}{c}n-1 \\ k\end{array}\right)+\gamma \delta\left(\begin{array}{c}n-1 \\ k-1\end{array}\right)\right]+n-2 \leq-1$, i.e.

$$
\gamma \geq(n-1)\left[\left(\begin{array}{c}
n-1 \\
k
\end{array}\right)+\delta\left(\begin{array}{l}
n-1 \\
k-1
\end{array}\right)\right]^{-1} .
$$

Comparing (5.11) and (5.12) we see that, in order to make the right-hand side finite and the left-hand side divergent, we must have

$$
\begin{aligned}
p & <\gamma^{-1} \min \left\{k, \frac{n-1}{\delta}\right\} \leq(n-1)^{-1} \min \left\{k, \frac{n-1}{\delta}\right\}\left[\left(\begin{array}{c}
n-1 \\
k
\end{array}\right)+\delta\left(\begin{array}{c}
n-1 \\
k-1
\end{array}\right)\right] \\
& =: g(\delta) \leq \max _{\delta>0} g(\delta) .
\end{aligned}
$$

Easy computations show that $g$ attains its maximum at $\delta=\frac{n-1}{k}$, for which we have

$$
p<g\left(\frac{n-1}{k}\right)=\left(\begin{array}{l}
n-2 \\
k-1
\end{array}\right)+\left(\begin{array}{l}
n-1 \\
k-1
\end{array}\right)=\tilde{p},
$$

thus proving the sharpness of the exponent $\tilde{p}$.

Remark 5.16. One could ask as in the case of functions of one variable if it is possible to obtain an inequality like (5.8) which is not a consequence of embeddings of $L^{p}\left(\mathbb{S}^{n-1}\right)$ spaces with a different $p_{i}$ for each $f_{i}$. In this case also an application of Theorem 3.10 is not effective. Indeed, even if one allows different $p_{i}$ 's in the nonlinear heat evolution associated to the operator $L$ defined in (5.4), the presence of functions of $k$ variables for each $k$-tuple of elements from $x_{1}, \ldots, x_{n}$ forces all exponents $p_{i}$ to be equal, since by symmetry each element of each finite set $\mathcal{A}_{i}^{c}$ has the same number $\tilde{p}$ of occurrences among the sets $\mathcal{A}_{k}^{c}$, for $k=1, \ldots, C(n, k)$.

Remark 5.17. Since constant functions are trivially functions of $k$ variables, inequality (5.8) also holds for $m \leq$ $C(n, k)$ functions of $m$ different $k$-tuples of variables. The inequality is nontrivial for $m>\tilde{p}$, since when $m \leq \tilde{p}$ a direct application of multilinear Hölder's inequality gives a better outcome in terms of exponents. Note that in this case an application of Theorem 3.10 could be effective. For example consider functions on the sphere $\mathbb{S}^{4}$ depending on 2 variables, for which $\tilde{p}=7$. There are 10 possible pairs of variables in the set $\{1, \ldots, 5\}$. Take just $m=8$ functions, say those associated to the pairs $(1,2),(1,3),(1,4),(1,5),(2,3),(2,4),(2,5),(3,4)$. We denote with $f_{i j}$ the nonnegative function depending on variables $x_{i}, x_{j}$ and with $\mathcal{A}_{i, j}^{c}$ the associated maximal set. It is easy to see that the vector field $L_{1,2}$ lies in all $\mathcal{A}_{i, j}^{c}$ except for $\mathcal{A}_{3,4}^{c}$. So the exponent associated to all functions except $f_{34}$ will be $\tilde{p}=7$. It is also easy to check that each element of $\mathcal{A}_{3,4}^{c}$ occurs at most six times among all the complements of the maximal sets. So an application of Theorem 3.10 shows that the inequality

$$
\int_{\mathbb{S}^{4}} f_{12} f_{13} f_{14} f_{15} f_{23} f_{24} f_{25} f_{34} d \sigma \leq\left\|f_{12}\right\|_{7}\left\|f_{13}\right\|_{7}\left\|f_{14}\right\|_{7}\left\|f_{15}\right\|_{7}\left\|f_{23}\right\|_{7}\left\|f_{24}\right\|_{7}\left\|f_{25}\right\|_{7}\left\|f_{34}\right\|_{6}
$$

holds. This inequality is nontrivial and is not a direct consequence of Theorem 5.14.

Remark 5.18. Thanks to Formula (5.2) it is possible to rewrite equation (5.9) in the form

$$
\int_{\mathbb{S}^{n-1}}^{C(n, k)} \prod_{i=1}^{C(n, k)} f_{i}\left(\pi_{\omega_{i}} x\right) d \sigma \lesssim \prod_{i=1}^{C(n}\left\|f_{i}\right\|_{L^{p}\left(B_{k}\right)},
$$

which has the structure of a Brascamp-Lieb inequality. 


\section{Further results}

\subsection{Inequalities for radial functions on $k$ variables}

In this section we improve on Theorem 5.14 by adding an additional symmetry. We consider functions of $k$ variables, i.e. functions that are defined on a $k$-dimensional unit ball and pulled-back to the sphere by means of a projection, that are radial with respect to the variables in the $k$-dimensional ball, for $1 \leq k \leq n-1$. Given a subset $\omega_{i}=\left\{i_{1}, \ldots, i_{k}\right\}$ of $\{1, \ldots, n\}$, we will use the notation $r\left(x_{\omega_{i}}\right)$ to denote the radius $\left(x_{i_{1}}^{2}+\cdots+x_{i_{k}}^{2}\right)^{1 / 2}$. A functions depending radially on the variables $x_{\omega}$ is a function $f:[0,1] \rightarrow \mathbb{R}$ pulled back to the sphere via the composition $r \circ \pi_{\omega_{i}}$. We will write $f\left(r\left(x_{\omega_{i}}\right)\right)$ for $f\left(\left(r\left(\pi_{\omega_{i}}(x)\right)\right)\right)$, with $x \in \mathbb{S}^{n-1}$.

We have $\left(\begin{array}{l}n \\ k\end{array}\right):=C(n, k)$ possible choices of $k$-tuples out of the set $\{1, \ldots, n\}$, as in the generic case of functions depending on $k$ variables. We will label the tuples by $\omega_{1}, \ldots, \omega_{C(n, k)}$, as in the previous section. To each collection of variables $\omega_{i}=\left\{i_{1}, \ldots, i_{k}\right\}$ corresponds a maximal subset $\mathcal{A}_{i}$ which contains all the vector fields $L_{h, l}$ for which $h, l \notin \omega_{i}$, but also the vector fields $L_{h, l}$ for which both $h, l \in \omega_{i}$, by the radiality assumption.

The subalgebra generated by each maximal subset $\mathcal{A}_{i}$ is isomorphic to the $\operatorname{direct} \operatorname{sum} \mathfrak{s o}(n-k) \oplus \mathfrak{s o}(k)$ and has the form $\left\langle\mathcal{A}_{i}\right\rangle=\mathfrak{s o}_{\alpha_{i}} \oplus \mathfrak{s o}_{\beta_{i}}$, where $\alpha_{i}$ is a multi-index such that $\alpha_{i}^{j}=0$ if $j \in \omega_{i}$ and $\beta_{i}=(1,1, \ldots, 1)-\alpha_{i}$.

Note that by the convention in Theorem 5.6 the splitting should be ordered by the cardinality of multiindices. We can reduce to the cases where $k \leq\left\lfloor\frac{n}{2} \mid\right.$. Indeed, consider a function $f$ that depends radially on the $k$ variables $\left\{x_{i_{1}}, \ldots, x_{i_{k}}\right\}$ and let $\left\{x_{i_{k+1}}, \ldots, x_{i_{n}}\right\}$ be the remaining $n-k$ variables. It is straightforward that

$$
f\left(x_{i_{1}}^{2}+\cdots+x_{i_{k}}^{2}\right)=f\left(1-\left(x_{i_{k+1}}^{2}+\cdots+x_{i_{n}}^{2}\right)\right)=g\left(x_{i_{k+1}}^{2}+\cdots+x_{i_{n}}^{2}\right),
$$

for some function $g$. There is a correspondence between functions that depend radially on $k$ variables and functions that depend radially on $n-k$ variables. Indeed, the number of possible choices of $k$-tuples and $(n-k)$ tuples is the same, since $\left(\begin{array}{c}n \\ k\end{array}\right)=\left(\begin{array}{c}n \\ n-k\end{array}\right)$, for $k \leq\left\lfloor\frac{n}{2}\right\rfloor$. Moreover the splittings of the corresponding associated maximal subsets is the same up to change in the order of the direct summands.

We will stick to the convention that the first direct summand is related to the longest multi-index, so it suffices to look at the case $k \leq\left\lfloor\frac{n}{2}\right\rfloor$. The case of $n$ even and $k=n / 2$ is a bit different and will be treated separately.

We have the following theorem.

Theorem 6.1. Let $k<n / 2$. Let $f_{1}, \ldots, f_{C(n, k)}$ be nonnegative measurable functions, $f_{i}:[0,1] \rightarrow \mathbb{R}^{+}$. The inequality

$$
\int_{\mathbb{S}^{n-1}} f_{1}\left(r\left(x_{\omega_{1}}\right)\right) \ldots f_{C(n, k)}\left(r\left(x_{\omega_{C(n, k)}}\right)\right) d \sigma \leq \prod_{i=1}^{C(n, k)}\left\|f_{i}\right\|_{L^{p}\left(\mathbb{S}^{n-1}\right)}
$$

holds for

$$
p \geq \tilde{p}=2\left(\begin{array}{l}
n-2 \\
k-1
\end{array}\right) .
$$

Moreover inequality (6.1) is sharp in the sense of Definition 5.12.

Remark 6.2. The result of [9] is again recovered, since functions that depend radially on one variable are just even functions of one variable. Indeed, for $k=1$ we have $\tilde{p}=2$. Note that the exponent $\tilde{p}$ obtained for this type of functions is smaller than that obtained for generic functions of $k$ variables. This in particular implies that inequality (6.1) is nontrivial in the sense of Definition 2.9.

Proof. By Theorem 3.8, the exponent $\tilde{p}$ is given by the number of occurrences of the most recurrent element among the sets $\mathcal{A}_{i}^{c}$, for $i=1, \ldots, C(n, k)$. The elements of $\mathcal{A}_{i}^{c}$ are vector fields of type $L_{h, l}$ with exactly one among $h$ and $l$ in $\omega_{i}$. So an element $L_{h, l}$ will occur in all $\mathcal{A}_{i}^{c}$ associated to subsets $\omega_{i}$ containing either $h$ but 
not $l$, which are $\left(\begin{array}{c}n-2 \\ k-1\end{array}\right)$, or $l$ but not $h$, which are again $\left(\begin{array}{c}n-2 \\ k-1\end{array}\right)$. Altogether, each vector field $L_{h, l}$ will occur $2\left(\begin{array}{c}n-2 \\ k-1\end{array}\right)$ times among the $\mathcal{A}_{i}^{c}$, yielding the exponent $\tilde{p}$.

To prove that $\tilde{p}$ is sharp we construct an explicit counterexample. Consider functions $f_{i}:[0,1] \rightarrow \mathbb{R}^{+}$, of the form

$$
f_{i}\left(r\left(x_{\omega_{i}}\right)\right)=\left(x_{i_{1}}^{2}+\cdots+x_{i_{k}}^{2}\right)^{-\gamma / 2}+\left(1-x_{i_{1}}^{2}-\cdots-x_{i_{k}}^{2}\right)^{-\frac{\gamma(n-k)}{2 k}},
$$

where $\gamma$ is a positive constants to be determined.

We first compute the norms on the right-hand side. Without loss of generality we assume that $\omega=$ $\{1, \ldots, k\}$ and work with $f\left(x_{1}^{2}+\cdots+x_{k}^{2}\right)$. Let $p \geq 1$. We have

$$
\begin{aligned}
\|f\|_{L^{p}\left(\mathbb{S}^{n-1}\right)}^{p} & \lesssim \int_{\mathbb{S}^{n-1}}\left[\left(x_{1}^{2}+\cdots+x_{k}^{2}\right)^{-\gamma p / 2}+\left(1-x_{1}^{2}-\cdots-x_{k}^{2}\right)^{-\frac{\gamma(n-k) p}{2 k}}\right] d \sigma \\
& \lesssim \int_{B_{k}}\left[\left(x_{1}^{2}+\cdots+x_{k}^{2}\right)^{-\gamma p / 2}\right. \\
& \left.+\left(1-x_{1}^{2}-\cdots-x_{k}^{2}\right)^{-\frac{\gamma(n-k) p}{2 k}}\right]\left(1-x_{1}^{2}-\cdots-x_{k}^{2}\right)^{(n-k-2) / 2} d x_{1} \ldots d x_{k} \\
& \lesssim \int_{0}^{1} \rho^{-\gamma p+k-1}\left(1-\rho^{2}\right)^{(n-2-k) / 2}+\rho^{k-1}\left(1-\rho^{2}\right)^{-\frac{\gamma(n-k) p}{2 k}+\frac{n-k-2}{2}} d \rho,
\end{aligned}
$$

where we used the integration formula (5.1) and then passed to polar coordinates. This integral is finite if $\gamma p<k$.

We control the left-hand side of (6.1) by the trivial bounds $\left(x_{1}^{2}+\cdots+x_{k}^{2}\right)^{-\gamma} \geq\left(x_{1}^{2}+\cdots+x_{k}^{2}+x_{k+1}^{2}+\cdots+x_{n-1}^{2}\right)^{-\gamma}$, for terms not involving $x_{n}$, and $\left(1-x_{1}^{2}-\cdots-x_{n}^{2}\right)^{-\gamma} \geq\left(1-x_{n}^{2}\right)^{-\gamma}$, for terms involving $x_{n}$. We make this distinction to pass to polar coordinates in the hyperplane $\mathbb{R}^{n-1}$ with coordinates $x_{1}, \ldots, x_{n-1}$; on the sphere $\mathbb{S}^{n-1},\left|x_{n}\right|$ will then just be $\left(1-\rho^{2}\right)^{1 / 2}$.

There are $\left(\begin{array}{c}n-1 \\ k\end{array}\right)$ terms not involving $x_{n}$, and $\left(\begin{array}{c}n-1 \\ k-1\end{array}\right)$ involving it. For the functions not depending on $x_{n}$ we select the first summand of (6.2), for those depending on $x_{n}$ we select the second one. For the left-hand side we have:

$$
\begin{aligned}
& \int_{\mathbb{S}^{n-1}} \prod_{i=1}^{C(n, k)} f_{i}\left(r\left(x_{\omega_{i}}\right)\right) d \sigma \geq \int_{\mathbb{S}^{n-1}}\left(x_{1}^{2}+\cdots+x_{n-1}^{2}\right)^{-\frac{\gamma}{2}\left(\begin{array}{c}
n-1 \\
k
\end{array}\right)}\left(1-x_{n}^{2}\right)^{-\frac{\gamma}{2} \frac{n-k}{k}\left(\begin{array}{c}
n-1 \\
k-1
\end{array}\right)} d \sigma \\
& \geq \int_{0}^{1} \rho^{-\left(\gamma\left(\begin{array}{c}
n-1 \\
k
\end{array}\right)+\gamma \frac{n-k}{k}\left(\begin{array}{c}
n-1 \\
k-1
\end{array}\right)\right)+n-2} \frac{d \rho}{\sqrt{1-\rho^{2}}} .
\end{aligned}
$$

This integral diverges for

$$
\gamma \geq(n-1)\left[\left(\begin{array}{c}
n-1 \\
k
\end{array}\right)+\frac{n-k}{k}\left(\begin{array}{l}
n-1 \\
k-1
\end{array}\right)\right]^{-1} .
$$

Comparing the condition $\gamma p<k$ and (6.3) we see that the right-hand side is finite and the left-hand side divergent if

$$
p<k \gamma^{-1} \leq \frac{k}{n-1}\left[\left(\begin{array}{c}
n-1 \\
k
\end{array}\right)+\frac{n-k}{k}\left(\begin{array}{l}
n-1 \\
k-1
\end{array}\right)\right]=2\left(\begin{array}{l}
n-2 \\
k-1
\end{array}\right)=\tilde{p},
$$

thus proving the optimality of the exponent $\tilde{p}$.

In the case of $n$ even and functions depending radially on $k=n / 2$ variables, the splitting associated to a maximal subset is of type $\mathfrak{s o}(n / 2) \oplus \mathfrak{s o}(n / 2)$ so that there are two possible orderings. This corresponds to the fact that, given a subset $\omega_{i}=\left\{i_{i}, \ldots, i_{n / 2}\right\}$ of $\{1, \ldots, n\}$, the set $\left\{i_{(n / 2)+1}, \ldots, i_{n}\right\}$ being its complement, a function radial in the variables of $\omega_{i}$ is also radial in the variables of its complement, but in this case both sets have cardinality $n / 2$. So one needs to consider a family of (different) $k$-tuples $\omega_{i}$, for $i=1, \ldots, C(n, k) / 2$, with $\omega_{i} \cap \omega_{j} \neq \emptyset$ for all $i, j$. Different choices of families of subsets $\omega_{i}$ give equivalent types of functions. We have the following theorem. 
Theorem 6.3. Let $n>3$ be even and $k=n / 2$. Let $\omega_{i}$ be a family of $C(n, k) / 2$ different $k$-tuples such that $\omega_{i} \cap \omega_{j} \neq \emptyset$ for all $i, j$. Let $f_{1}, \ldots, f_{C(n, k) / 2}$ be nonnegative measurable functions, $f_{i}:[0,1] \rightarrow \mathbb{R}^{+}$. The inequality

$$
\int_{\mathbb{S}^{n-1}} f_{1}\left(r\left(x_{\omega_{1}}\right)\right) \ldots f_{C(n, k) / 2}\left(r\left(x_{\omega_{C(n, k) / 2}}\right)\right) d \sigma \leq \prod_{i=1}^{C(n, k) / 2}\left\|f_{i}\right\|_{L^{p}\left(\mathbb{S}^{n-1}\right)}
$$

holds for

$$
p \geq \tilde{p}=\left(\begin{array}{l}
n-2 \\
k-1
\end{array}\right) .
$$

Moreover inequality (6.4) is sharp in the sense of Definition 5.12.

Proof. We need to compute the number of occurrences of each vector field $L_{h, l}$ among the sets $\mathcal{A}_{i}^{c}$. As before we must consider sets $\omega_{i}$ containing either $h$ or $l$, but not both. To a $k$-tuple $\sigma$ containing $h$ and not $l$ corresponds a unique $k$-tuple $\tau$ containing $l$ and not $h$ such that $\sigma \cap \tau=\emptyset$. By the assumptions on the $\omega_{i}$, one and just one between $\sigma$ and $\tau$ is among the sets $\omega_{i}$. Thus, each vector field $L_{h, l}$ occurs $\left(\begin{array}{c}n-2 \\ k-1\end{array}\right)$ times among the $\mathcal{A}_{i}^{c}$. This provides the exponent $\tilde{p}$.

The sharpness is proved as in Theorem 6.1, by testing on the functions

$$
f_{i}\left(r\left(x_{\omega_{i}}\right)\right)=\left(x_{i_{1}}^{2}+\cdots+x_{i_{k}}^{2}\right)^{-(n-1) / C(n, k)} .
$$

\subsection{Inequalities with different exponents}

In Remark 5.16 of Theorem 5.14 we saw that an application of Theorem 3.8 always yields the same exponent for all functions. In this section we want to understand for which exponents $p_{1}, \ldots, p_{C(n, k)}$ an inequality of the type

$$
\int_{\mathbb{S}^{n-1}} \prod_{i=1}^{C(n, k)} f_{i}\left(x_{\omega_{i}}\right) d \sigma \leq \prod_{i=1}^{C(n, k)}\left\|f_{i}\right\|_{L^{p_{i}\left(\mathbb{S}^{n-1}\right)}}
$$

may hold. Since $p_{i}^{-1}$ belongs to $[0,1]$, only a point in the unit cube $Q=[0,1]^{C(n, k)}$ in $\mathbb{R}^{C(n, k)}$ identifies a choice of exponents. The inequality holds for points $\left(p_{1}^{-1}, \ldots, p_{C(n, k)}^{-1}\right) \in Q$ for which

$$
\sum_{i=1}^{C(n, k)} p_{i}^{-1} \leq 1
$$

by Hölder's inequality and continuous embeddings of Lebesgue spaces. By Theorem 5.14 we know that (6.5) also holds for $\left(\tilde{p}^{-1}, \ldots, \tilde{p}^{-1}\right) \in Q$, with $\tilde{p}=\left(\begin{array}{c}n \\ k\end{array}\right)-\left(\begin{array}{c}n-2 \\ k\end{array}\right)$. Then inequality (6.5) holds for points $\left(p_{1}^{-1}, \ldots, p_{C(n, k)}^{-1}\right) \in \mathcal{Q}$ for which

$$
\sum_{i=1}^{C(n, k)} p_{i}^{-1} \leq \frac{C(n, k)}{\tilde{p}}
$$

with $p_{i}>\tilde{p}$ for all $i=1, \ldots, C(n, k)$, by continuous embeddings of Lebesgue spaces again. Nevertheless we can extend the range of exponents for which (6.5) is valid applying multilinear interpolation (see [19] or [12]) to the operator

$$
T\left(f_{1}, \ldots, f_{C(n, k)}\right)=\int_{\mathbb{S}^{n-1}} \prod f_{i}\left(\pi_{\omega_{i}} x\right) d \sigma,
$$

where the functions $f_{i}$ are defined on the measure space $\left(B_{k},\left(1-|x|^{2}\right)^{(n-k-2) / 2} d x\right)$ and $d x$ is the Lebesgue measure in $\mathbb{R}^{k}$. Recall that thanks to the integration formula (5.1) we have

$$
\left\|f_{i} \circ \pi_{\omega_{i}}\right\|_{L^{p}\left(\mathbb{S}^{n-1}\right)} \simeq\left\|f_{i}\right\|_{L^{p}\left(B_{k},\left(1-|x|^{2}\right)^{(n-k-2) / 2} d x\right)} .
$$


By interpolating the exponents that verify Hölder's condition with $\left(\tilde{p}^{-1}, \ldots, \tilde{p}^{-1}\right)$, we obtain the following proposition.

Proposition 6.4. Let $\left(p_{1}^{-1}, \ldots, p_{C(n, k)}^{-1}\right) \in Q$ be such that $\sum_{i=1}^{C(n, k)} p_{i}^{-1} \leq 1$. Then the inequality

$$
\int_{\mathbb{S}^{n-1}} \prod_{i=1}^{C(n, k)} f_{i}\left(x_{\omega_{i}}\right) d \sigma \leq \prod_{i=1}^{C(n, k)}\left\|f_{i}\right\|_{L^{r_{i}}\left(\mathbb{S}^{n-1}\right)}
$$

holds for all exponents $r_{i}$ such that $r_{i}^{-1}=\theta p_{i}^{-1}+(1-\theta) \tilde{p}^{-1}$ for all $i=1, \ldots, C(n, k)$ and $\theta \in[0,1]$.

So (6.5) holds in the convex hull $\mathcal{R}$ of the region $\sum p_{i}^{-1} \leq 1$ and the point $\left(\tilde{p}^{-1}, \ldots, \tilde{p}^{-1}\right)$. We conjecture that outside $\mathcal{R}$ the inequality (6.5) is false. Theorem 5.14 excludes the points $\left(p_{1}^{-1}, \ldots, p_{C(n, k)}^{-1}\right) \in \mathcal{Q}$ such that $p_{i}<\tilde{p}$ for all $i$, that are not in $\mathcal{R}$, but do not exhaust the complement of $\mathcal{R}$ in $\mathcal{Q}$.

Unfortunately we do not have a complete proof of the conjecture. We have however the following partial result for points in the hyperplane in $Q$ given by the equation

$$
\sum_{i=1}^{C(n, k)} p_{i}^{-1}=\frac{C(n, k)}{\tilde{p}},
$$

to which $\left(\tilde{p}^{-1}, \ldots, \tilde{p}^{-1}\right)$ belongs.

Theorem 6.5. Let $\left(p_{1}^{-1}, \ldots, p_{C(n, k)}^{-1}\right) \in \mathcal{Q}$ be such that

$$
\sum_{i=1}^{C(n, k)} p_{i}^{-1}=\frac{C(n, k)}{\tilde{p}} .
$$

For any $l$ consider the set $J_{l}$ consisting of the indices $j$ such that $l \in \omega_{j}\left(\right.$ then $\left.\left|J_{l}\right|=\left(\begin{array}{c}n-1 \\ k-1\end{array}\right)\right)$. If there is $l$ such that

$$
\sum_{j \in J_{l}} p_{j}^{-1}>\frac{\left(\begin{array}{c}
n-1 \\
k-1
\end{array}\right)}{\tilde{p}},
$$

then the inequality (6.5) is false.

Proof. Without loss of generality suppose that $l=n$ in the hypothesis. We label the $\left(\begin{array}{l}n-1 \\ k-1\end{array}\right)$ sets $\omega_{i}$ for which $n \in \omega_{i}$ with $i=1, \ldots,\left(\begin{array}{c}n-1 \\ k-1\end{array}\right)$. Consider functions $f_{i}: B_{k} \rightarrow \mathbb{R}^{+}$, where $B_{k}$ is the unit ball in $\mathbb{R}^{k}$, of the form

$$
f_{i}\left(x_{\omega_{i}}\right)=\left(\left|x_{i_{1}}\right|\left|x_{i_{2}}\right| \ldots \mid x_{i_{k}}\right)^{-\gamma_{i} / k}+\left(1-x_{i_{1}}^{2}\right)^{-\frac{\gamma_{i}(n-1)}{2 k}}+\cdots+\left(1-x_{i_{k}}^{2}\right)^{-\frac{\gamma_{i}(n-1)}{2 k}} .
$$

From the proof of Theorem 5.14 we know that each $f_{i}$ is in $L^{p_{i}}\left(\mathbb{S}^{n-1}\right)$ if

$$
\gamma_{i} p_{i}<k
$$

for all $i=1, \ldots, C(n, k)$, so that under this assumption the right-hand side of (6.5) is finite.

For the left-hand side we proceed as follows. For the functions not depending on the variable $x_{n}$ we select the first summand in (6.8), for those depending on $x_{n}$ we select the summand $\left(1-x_{n}^{2}\right)^{-\frac{\gamma_{i}(n-1)}{2 k}}$. So the left-hand side satisfies:

$$
\begin{aligned}
\int_{\mathbb{S}^{n-1}} \prod_{i=1}^{C(n, k)} f_{i}\left(x_{\omega_{i}}\right) d \sigma & \geq \int_{\mathbb{S}^{n-1}} \prod_{i=\left(\begin{array}{c}
n-1 \\
k-1
\end{array}\right)+1}^{C(n, k)}\left(\left|x_{i_{1}}\right| \ldots\left|x_{i_{k}}\right|\right)^{-\frac{\gamma_{i}}{k}} \prod_{j=1}^{\left(\begin{array}{l}
n-1 \\
k-1
\end{array}\right)}\left(1-x_{n}^{2}\right)^{-\frac{\gamma_{j}(n-1)}{2 k}} d \sigma \\
& \geq \int_{0}^{1} \rho^{-\sum_{i} \gamma_{i}-\frac{n-1}{k} \sum_{j} \gamma_{j}+n-2} \frac{d \rho}{\sqrt{1-\rho^{2}}},
\end{aligned}
$$


where we proceeded as in the proof of Theorem 5.14. Set $I=\left\{\left(\begin{array}{c}n-1 \\ k-1\end{array}\right)+1, \ldots, C(n, k)\right\}$ and $J=\left\{1, \ldots,\left(\begin{array}{c}n-1 \\ k-1\end{array}\right)\right\}$. The left-hand side of (6.5) diverges if

$$
-\sum_{i \in I} \gamma_{i}-\frac{n-1}{k} \sum_{j \in J} \gamma_{j}+n-2=-1
$$

that is if

$$
\sum_{i \in I} \gamma_{i}=(n-1)\left(1-\frac{1}{k} \sum_{j \in J} \gamma_{j}\right) .
$$

Since by (6.9) $\sum_{i \in I} \gamma_{i}<k \sum_{i \in I} p_{i}^{-1}$, to make the left-hand side divergent we must have

$$
\left((n-1)-k \sum_{i \in I} p_{i}^{-1}\right)<\frac{n-1}{k} \sum_{j \in J} \gamma_{j} .
$$

Since by (6.9) $\sum_{j \in J} \gamma_{j}<k \sum_{j \in J} p_{j}^{-1}$, we must also have

$$
\left(1-\frac{k}{n-1} \sum_{i \in I} p_{i}^{-1}\right)<\frac{1}{k} \sum_{j \in J} \gamma_{j}<\sum_{j \in J} p_{j}^{-1} .
$$

It is possible to choose $\gamma_{j}$ so that $k^{-1} \sum_{j \in J} \gamma_{j}$ is squeezed between these two terms if

$$
\left(1-\frac{k}{n-1} \sum_{i \in I} p_{i}^{-1}\right)<\sum_{j \in J} p_{j}^{-1}
$$

which by (6.6) becomes

$$
1-\frac{k}{n-1}\left(\frac{C(n, k)}{\tilde{p}}-\sum_{j \in J} p_{j}^{-1}\right)<\sum_{j \in J} p_{j}^{-1},
$$

which is equivalent to

$$
\sum_{j \in J} p_{j}^{-1}>\left(1-\frac{k}{n-1}\right)^{-1}\left(1-\frac{k}{n-1} \frac{C(n, k)}{\tilde{p}}\right),
$$

from which the assertion follows.

Remark 6.6. Note that there are $n$ and $k$ for which the assumptions of Theorem 6.5 are not fulfilled. For example, consider $n=4$ and $k=2$, i.e. the case of functions of two variables on the sphere $\mathbb{S}^{3}$, for which $\tilde{p}=5$. There are 6 possible tuples $(i, j)$, with $1 \leq i<j \leq 4$. We will denote by $p_{i j}$ the exponent corresponding to the pair $(i, j)$. It is easy to check that choosing $p_{12}=p_{13}=p_{24}=p_{34}=10, p_{23}=p_{14}=5 / 2$, we have that $\sum_{i<j} p_{i j}^{-1}=6 / 5$. Nevertheless, Theorem 6.5 cannot be applied, since for all $l$ and all triples $\left(j_{1}, j_{2}, j_{3}\right)$ we have $p_{l j_{1}}+p_{l j_{2}}+p_{l j_{3}} \leq 3 / 5$, so that (6.7) is never satisfied.

\subsection{The case $n=3$ and $k=1$}

In this section we will discuss in more detail the case of functions of one variable on the sphere $\mathbb{S}^{2}$. We want to understand for which $\left(p_{1}^{-1}, p_{2}^{-1}, p_{3}^{-1}\right) \in \mathcal{Q}=[0,1]^{3}$ the inequality

$$
\int_{\mathbb{S}^{2}} f_{1}\left(x_{1}\right) f_{2}\left(x_{2}\right) f_{3}\left(x_{3}\right) d \sigma \leq\left\|f_{1}\right\|_{L^{p_{1}}\left(\mathbb{S}^{2}\right)}\left\|f_{2}\right\|_{L^{p_{2}\left(\mathbb{S}^{2}\right)}}\left\|f_{3}\right\|_{L^{p_{3}}\left(\mathbb{S}^{2}\right)}
$$

holds true for all measurable functions $f_{i}:[-1,1] \rightarrow \mathbb{R}^{+}$, for $i=1,2,3$. As explained in the previous section, the inequality holds in the region $\mathcal{R}$, which is the convex hull of the the Hölder's tetrahedron and 
the point $(1 / 2,1 / 2,1 / 2)$ given by Theorem 5.13. Moreover in this case the assumptions of Theorem 6.5 are always fulfilled, since given any triple $\left(p_{1}^{-1}, p_{2}^{-1}, p_{3}^{-1}\right) \neq(1 / 2,1 / 2,1 / 2)$ such that $p_{1}^{-1}+p_{2}^{-1}+p_{3}^{-1}=3 / 2$, by pigeonholing there must always be one $p_{i}>1 / 2$. This implies that the point $(1 / 2,1 / 2,1 / 2)$ is the only point in the hyperplane $p_{1}^{-1}+p_{2}^{-1}+p_{3}^{-1}=3 / 2$ where inequality (6.10) holds. From this we also deduce that inequality (6.10) cannot hold for points in $Q$ such that $p_{1}^{-1}+p_{2}^{-1}+p_{3}^{-1}>3 / 2$. Indeed, by interpolation with points in $\mathcal{R}$ this would yield points in the hyperplane $p_{1}^{-1}+p_{2}^{-1}+p_{3}^{-1}=3 / 2$ for which the inequality holds, providing a contradiction. This goes in the direction of our conjecture, that $\mathcal{R}$ is the optimal region of validity for (6.10).

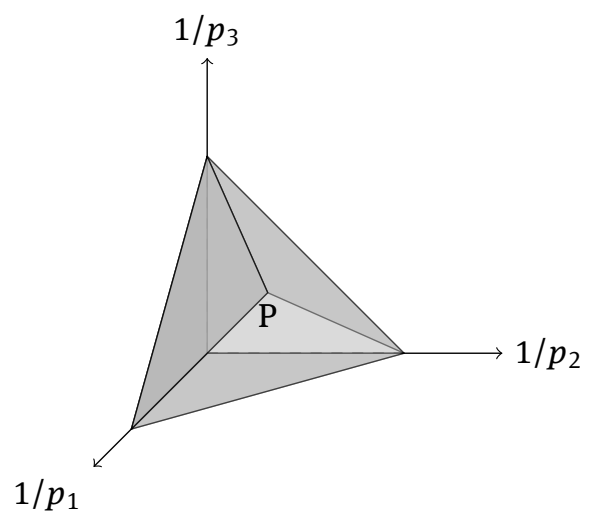

Figure 1: The conjectured sharp region $\mathcal{R}$, where $P=(1 / 2,1 / 2,1 / 2)$.

The only points left are those outside of $\mathcal{R}$ for which $1<p_{1}^{-1}+p_{2}^{-1}+p_{3}^{-1}<3 / 2$. In this range we have the following proposition which leads to a partial improvement towards the sharpness.

Proposition 6.7. Suppose that $1<p_{1}^{-1}+p_{2}^{-1}+p_{3}^{-1}<3 / 2$ and that the condition

$$
\frac{1}{p_{a}}+\frac{1}{p_{b}}>2\left(1-\frac{1}{p_{c}}\right)
$$

holds for at least one choice of $a, b, c$ in $\{1,2,3\}$ with $a, b, c$ pairwise distinct. Then inequality (6.10) is false.

Proof. We make the usual construction. Assume for instance that $a=1, b=2, c=3$. We let

$$
f_{i}\left(x_{i}\right)=\left|x_{i}\right|^{-\gamma_{i}}+\left(1-x_{i}^{2}\right)^{-\frac{(n-1) \gamma_{i}}{2}}=\left|x_{i}\right|^{-\gamma_{i}}+\left(1-x_{i}^{2}\right)^{-\gamma_{i}},
$$

for $i=1,2,3$. As usual the integrability condition for the right-hand side of (6.10) is $\gamma_{i} p_{i}<1$. For the left-hand side, taking the first summand for $f_{1}$ and $f_{2}$ and the second one for $f_{3}$, we get that

$$
\int_{\mathbb{S}^{2}} f_{1}\left(x_{1}\right) f_{2}\left(x_{2}\right) f_{3}\left(x_{3}\right) d \sigma \geq \int_{0}^{1} \rho^{-\gamma_{1}-\gamma_{2}-2 \gamma_{3}+1} \frac{d \rho}{\sqrt{1-\rho^{2}}},
$$

which diverges for $\gamma_{1}+\gamma_{2}+2 \gamma_{3}=2$, that is for

$$
\gamma_{3}=1-\frac{\gamma_{1}+\gamma_{2}}{2} .
$$

From the condition $\gamma_{i} p_{i}<1$ we get that we need to have

$$
2\left(1-\frac{1}{p_{3}}\right)<\gamma_{1}+\gamma_{2}<\frac{1}{p_{1}}+\frac{1}{p_{2}} .
$$

Clearly $\gamma_{1}+\gamma_{2}$ can be in this range only when (6.11) holds.

To sum up, we do not know what happens in the range $1<p_{1}^{-1}+p_{2}^{-1}+p_{3}^{-1}<3 / 2$, outside of $\mathcal{R}$, where none of the conditions (6.11) is satisfied for any exponent $p_{i}$. An example of a point in this region is $(2 / 3,2 / 3,0)$. 


\subsection{Inequalities with other symmetries}

In the last sections we saw applications of Theorem 3.8 in special cases, where the choices of the maximal subsets $\mathcal{A}_{i}$ of $\left\{L_{j, l}\right\}_{j<l}$ reflected particular symmetries of the functions involved. Nevertheless, Theorem 3.8 (and Theorem 3.10) can also be applied to other type of symmetries. Indeed, let $\mathcal{A}_{i}$ be maximal subsets for $i=1, \ldots, m$.

An easy algorithm to compute the exponent $\tilde{p}$ of Theorem 3.8 and the exponents $\tilde{p}_{i}$ of Theorem 3.10 is as follows. We consider the matrix of zeros and ones with $m$ rows indexed by the $m$ maximal subsets and $\left(\begin{array}{l}n \\ 2\end{array}\right)$ columns indexed by the vector fields of the basis of $\mathfrak{s o}(n)$. We set $a_{i j}=1$ if the vector field corresponding to the $j$-th column is in $\mathcal{A}_{i}^{c}$ and zero otherwise. In this way the exponent $\tilde{p}$ of Theorem 3.8, being the number of occurrences of the most recurrent element among the $\mathcal{A}_{i}^{c}$, is just

$$
\max _{j} \sum_{i=1}^{m} a_{i j}
$$

The exponent $\tilde{p}_{i}$ in Theorem 3.10, being the number of occurrences of the most recurrent element in $\mathcal{A}_{i}^{c}$, is given by

$$
\max _{j: a_{i j}=1} \sum_{k=1}^{m} a_{k j}
$$

where we take the maximum only over the columns $j$ for which $a_{i j}=1$, so that we check how many times the vector fields that are contained in $\mathcal{A}_{i}^{c}$ occur in the sets $\mathcal{A}_{k}^{c}$.

We give two examples. We remark that also in these examples the exponents given by Theorem 3.8 turn out to be sharp.

Example 6.8. On the sphere $\mathbb{S}^{3}$ consider three functions, $f_{1}$ depending on $x_{1}, f_{2}$ depending on $x_{2}$, and $f_{3}$ depending radially on $x_{1}$ and $x_{2}$ (or equivalently depending radially on $x_{3}$ and $x_{4}$ ). The maximal subset annihilating $f_{1}$ is $\mathcal{A}_{1}=\left\{L_{2,3}, L_{2,4}, L_{3,4}\right\}$, with $\left\langle\mathcal{A}_{1}\right\rangle \simeq \mathfrak{s o}(3)$, so that $\mathcal{A}_{1}^{c}=\left\{L_{1,2}, L_{1,3}, L_{1,4}\right\}$. The maximal subset annihilating $f_{2}$ is $\mathcal{A}_{2}=\left\{L_{1,3}, L_{1,4}, L_{3,4}\right\}$, with $\left\langle\mathcal{A}_{2}\right\rangle \simeq \mathfrak{s o}(3)$, so that $\mathcal{A}_{2}^{c}=\left\{L_{1,2}, L_{2,3}, L_{2,4}\right\}$. The maximal subset annihilating $f_{3}$ is $\mathcal{A}_{3}=\left\{L_{1,2}, L_{3,4}\right\}$, with $\left\langle\mathcal{A}_{3}\right\rangle \simeq \mathfrak{s o}(2) \oplus \mathfrak{s o}(2)$, so that $\mathcal{A}_{3}^{c}=\left\{L_{1,3}, L_{1,4}, L_{2,3}, L_{2,4}\right\}$. Each $\mathcal{A}_{i}^{c}$ has an element that occurs twice among the sets $\mathcal{A}_{k}^{c}$, for $k=1,2$, 3, so by Theorem 3.8 we have $\tilde{p}=2$. It follows that

$$
\int_{\mathbb{S}^{3}} f_{1}\left(x_{1}\right) f_{2}\left(x_{2}\right) f_{3}\left(x_{1}^{2}+x_{2}^{2}\right) d \sigma \leq\left\|f_{1}\right\|_{L^{2}\left(\mathbb{S}^{3}\right)}\left\|f_{2}\right\|_{L^{2}\left(\mathbb{S}^{3}\right)}\left\|f_{3}\right\|_{L^{2}\left(\mathbb{S}^{3}\right)} .
$$

Moreover this inequality is sharp. Indeed, consider the functions $f_{i}\left(x_{i}\right)=\left|x_{i}\right|^{-1 / 2}$ for $i=1,2$ and the function $f_{3}\left(x_{1}^{2}+x_{2}^{2}\right)=\left(x_{1}^{2}+x_{2}^{2}\right)^{-1 / 2}$. It is easy to see, proceeding in the same way as above, that $\left\|f_{i}\right\|_{L^{p}\left(\mathbb{S}^{3}\right)}<\infty$ with $p<2$ for $i=1,2,3$. Nonetheless we have that

$$
\begin{aligned}
\int_{\mathbb{S}^{3}} f_{1} f_{2} f_{3} d \sigma & =\int_{\mathbb{S}^{3}}\left|x_{1}\right|^{-1 / 2}\left|x_{2}\right|^{-1 / 2}\left(x_{1}^{2}+x_{2}^{2}\right)^{-1 / 2} d \sigma \\
& \sim \int_{B_{2}}\left|x_{1}\right|^{-1 / 2}\left|x_{2}\right|^{-1 / 2}\left(x_{1}^{2}+x_{2}^{2}\right)^{-1 / 2}\left(1-x_{1}^{2}-x_{2}^{2}\right)^{(4-2-2) / 2} d x_{1} d x_{2} \\
& \gtrsim \int_{0}^{1} \rho^{-(1 / 2)-(1 / 2)-1} \rho d \rho=\int_{0}^{1} \rho^{-1} d \rho,
\end{aligned}
$$

that diverges.

Example 6.9. On the sphere $\mathbb{S}^{4}$ we consider functions depending on three variables, with radial dependence on two of them. This corresponds to the case of maximal subsets $\mathcal{A}_{i}$ with two elements $L_{i, j}, L_{k, l}$ with $i, j, k, l$ pairwise distinct so that the generated subalgebras have the form $\left\langle\mathcal{A}_{i}\right\rangle=\mathfrak{s o}(2) \oplus \mathfrak{s o}(2)$. As we discussed above, the first subalgebra indicates the number of variables the functions depend on, in this case we have 
$n-k=5-2=3$. The second subalgebra refers to radiality in two of the variables involved. The ambiguity in the order of the subalgebras is not a problem, since the two possibilities are equivalent in the following sense. If $\mathcal{A}=\left\{L_{1,2}, L_{3,4}\right\}$ we are considering a function $f$ either of type $f\left(x_{3}^{2}+x_{4}^{2}, x_{5}\right)$ or a function of type $f\left(x_{1}^{2}+x_{2}^{2}, x_{5}\right)$ which are equivalent, since $x_{3}^{2}+x_{4}^{2}=1-x_{1}^{2}-x_{2}^{2}-x_{5}^{2}$. There are $\left(\begin{array}{l}5 \\ 2\end{array}\right)=10$ possible choices for $L_{i, j}$, and having fixed $i$ and $j$ we have $\left(\begin{array}{l}3 \\ 2\end{array}\right)=3$ choices for $L_{k, l}$. By the aforementioned equivalence we have 15 possible maximal subsets.

It is easy to see that in this case the critical exponent given by Theorem 3.8 is $\tilde{p}=12$. The exponent is sharp and this can be easily checked considering the functions

$$
f_{i}^{j l}\left(x_{i}, x_{j}^{2}+x_{l}^{2}\right)=\left|x_{i}\right|^{-1 / 12}\left(x_{j}^{2}+x_{l}^{2}\right)^{-1 / 12}+\left(1-x_{i}^{2}\right)^{-1 / 6} .
$$

Acknowledgements: Part of the research contained in this paper was carried out while visiting the School of Mathematics at The University of Edinburgh. I would like to thank Prof. Anthony Carbery and Prof. James Wright for interesting discussions and remarks and the School of Mathematics for the kind hospitality.

\section{References}

[1] K. Ball, Volumes of sections of cubes and related problems, Geometric aspects of functional analysis (1987-88), Lecture Notes in Math., vol. 1376, 251-260, Springer, Berlin, 1989.

[2] F. Barthe, On a reverse form of the Brascamp-Lieb inequality, Invent. Math., 134(2):335-361, 1998.

[3] F. Barthe, D. Cordero-Erausquin, M. Ledoux, and B. Maurey, Correlation and Brascamp-Lieb inequalities for Markov semigroups, Int. Math. Res. Not. IMRN, (10):2177-2216, 2011.

[4] F. Barthe, D. Cordero-Erausquin, and B. Maurey, Entropy of spherical marginals and related inequalities, J. Math. Pures Appl. (9), 86(2):89-99, 2006

[5] J. Bennett, A. Carbery, M. Christ, and T. Tao, The Brascamp-Lieb inequalities: finiteness, structure and extremals, Geom. Funct. Anal., 17(5):1343-1415, 2008.

[6] H. J. Brascamp and E. H. Lieb, Best constants in Young's inequality, its converse, and its generalization to more than three functions, Advances in Math., 20(2):151-173, 1976.

[7] H. J. Brascamp, E. H. Lieb, and J. M. Luttinger, A general rearrangement inequality for multiple integrals, J. Functional Analysis, 17:227-237, 1974

[8] A. P. Calderón, An inequality for integrals, Studia Math., 57(3):275-277, 1976.

[9] E. A. Carlen, E. H. Lieb, and M. Loss, A sharp analog of Young's inequality on $S^{N}$ and related entropy inequalities, J. Geom. Anal., 14(3):487-520, 2004.

[10] E. A. Carlen, E. H. Lieb, and M. Loss, An inequality of Hadamard type for permanents, Methods Appl. Anal., 13(1):1-17, 2006.

[11] H. Finner, A generalization of Hölder's inequality and some probability inequalities, Ann. Probab., 20(4):1893-1901, 1992.

[12] L. Grafakos, Classical Fourier analysis, Graduate Texts in Mathematics, vol. 249, Springer, New York, third edition, 2014.

[13] S. Helgason, Differential geometry and symmetric spaces, Pure and Applied Mathematics, Vol. XII, Academic Press, New York-London, 1962.

[14] G. A. Hunt, Semi-groups of measures on Lie groups, Trans. Amer. Math. Soc., 81:264-293, 1956.

[15] E. H. Lieb, Gaussian kernels have only Gaussian maximizers, Invent. Math., 102(1):179-208, 1990.

[16] L. H. Loomis and H. Whitney, An inequality related to the isoperimetric inequality, Bull. Amer. Math. Soc, 55:961-962, 1949.

[17] P. Maheux, Estimations du noyau de la chaleur sur les espaces homogènes, J. Geom. Anal., 8(1):65-96, 1998.

[18] E. Nelson, Analytic vectors, Ann. of Math. (2), 70:572-615, 1959.

[19] G. O. Okikiolu, A convexity theorem for multilinear functionals, J. Math. Anal. Appl., 16:165-172, 1966.

[20] C. A. Rogers, Two integral inequalities, J. London Math. Soc., 31:235-238, 1956.

[21] E. M. Stein and G. Weiss, Introduction to Fourier analysis on Euclidean spaces, Princeton University Press, Princeton, N.J., 1971.

[22] N. T. Varopoulos, L. Saloff-Coste, and T. Coulhon, Analysis and geometry on groups, Cambridge Tracts in Mathematics, vol. 100, Cambridge University Press, Cambridge, 1992. 